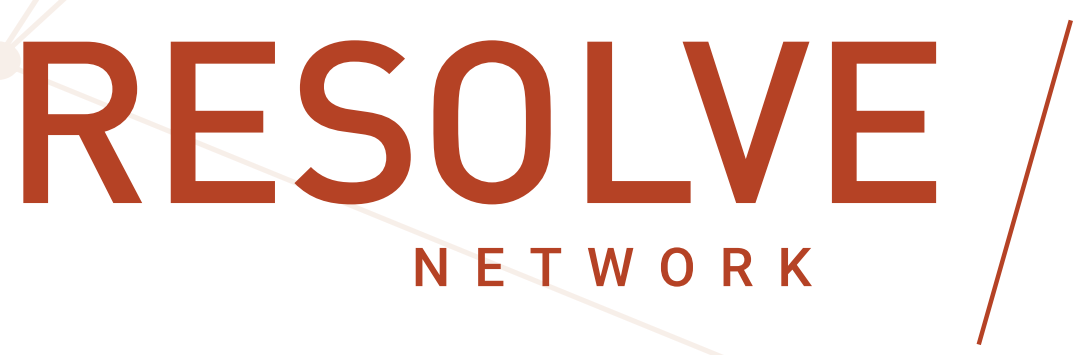

\title{
Une approche
} des groupes armés communautaires en Afrique subsaharienne Enseignements tirés et mesures de la réussite Dr Moritz Schuberth 


\section{CONTENU}

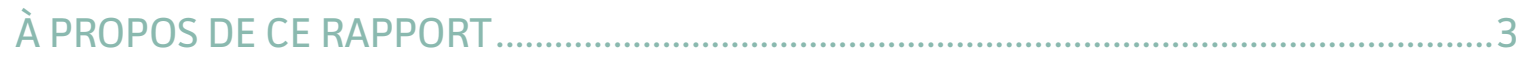

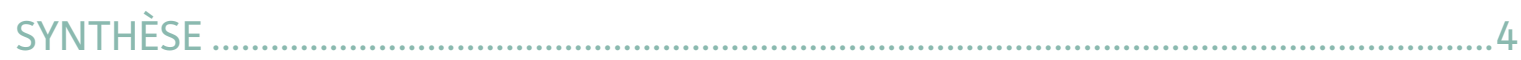

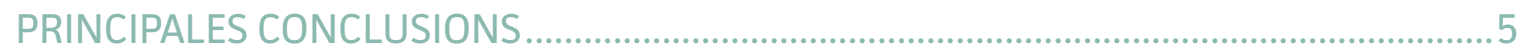

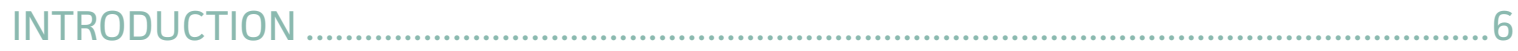

REMISE EN CAUSE DES HYPOTHĖSES COMMUNES...............................................8

CARTOGRAPHIE DES APPROCHES EN MATIÈRE D'EGT DES GAC

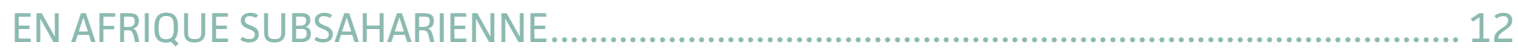

ENSEIGNEMENTS TIRÉS DES APPROCHES EN MATIÈRE D’EGT DES GAC ................... 17

TENDANCES ACTUELLES ET NOUVELLES ORIENTATIONS ........................................ 24

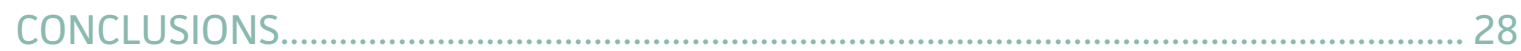

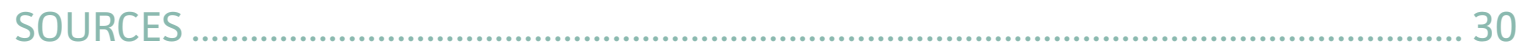




\section{À PROPOS DE CE RAPPORT}

Ce rapport a été documenté et rédigé par le Dr Moritz Schuberth. Un certain nombre de membres du secrétariat du Réseau RESOLVE ont contribué à l'élaboration de ce rapport, notamment Mme Bethany McGann, responsable recherche et projet ; Mme Boglarka Bozsogi, coordinatrice de la recherche et des communications; Mme Kateira Aryaeinejad, responsable de la recherche et du projet; et Mme Leanne Erdberg, directrice exécutive par intérim. RESOLVE tient à remercier les réviseurs de ce rapport et les membres du Conseil consultatif de la recherche du Réseau RESOLVE de lui avoir apporté leur soutien et leurs conseils. Enfin, RESOLVE tient à remercier le bureau africain de la United States Agency for International Development (USAID) pour son généreux soutien à ce rapport et à l'initiative de recherche de RESOLVE sur les groupes armés communautaires en Afrique subsaharienne.

Dr Schuberth est un politologue ayant un penchant pour la recherche interdisciplinaire, notamment sur le maintien de la paix, les groupes armés non étatiques, la gouvernance de la sécurité et la violence urbaine. Depuis deux ans, il travaille en tant que responsable de l'évaluation et de la recherche pour l'agence humanitaire internationale Mercy Corps dans l'est de la République démocratique du Congo, où il coordonne des projets de recherche avec l'université de Harvard, l'université de Cornell et la London School of Economics. Auparavant, il a travaillé à la Commission européenne, au Bureau fédéral allemand des affaires étrangères et à l'Office fédéral allemand des migrations et des réfugiés.

Dr Schuberth a obtenu son doctorat en études sur la paix et les conflits en s'intéressant au défi de la coordination des efforts de stabilisation, de résolution des conflits et de renforcement de l'État dans les États fragiles et touchés par les conflits. Il a contribué à un livre blanc du ministère de la Défense américain sur les implications de la diversité cognitive culturelle sur la prise de décisions et la planification, et ses recherches ont été utilisées pour former les forces spéciales américaines de résolution des conflits. II est l'auteur de récents articles dans Africa Spectrum; le Journal of Eastern African Studies, Conflict, Security \& Development ; le Journal of Peacebuilding and Development ; Contemporary Security Policy ; Stability: International Journal of Security and Development ; International Peacekeeping ; et Environment and Urbanization.

Une approche des groupes armés communautaires en Afrique subsaharienne fait partie de la série de recherches sur les groupes armés communautaires du Réseau RESOLVE, une initiative qui étudie la dynamique des groupes armés communautaires et les contextes dans lesquels ils opèrent pour identifier des approches potentielles pour les engager, les gérer et les transformer.

Pour plus d'informations sur RESOLVE, son réseau d'experts, ses projets et activités de recherche, veuillez visiter notre site Web à l'adresse www.resolvenet.org et suivre la discussion sur Twitter via @resolvenet. 


\section{SYNTHÈSE}

\section{Objectif de cette étude}

L'augmentation de la prévalence des groupes armés communautaires ou GAC (community-based armed groups ou CBAGs) en Afrique subsaharienne fait craindre qu'ils posent un défi croissant aux gouvernements et aux citoyens de la région. Ce document cartographie la manière dont les différents acteurs intervenants (communautés, gouvernements, organisations de la société civile, agences humanitaires et de développement et fournisseurs de sécurité) ont abordé les défis posés par les GAC et comment mesurer au mieux le succès de ces interventions. La cartographie des approches, l'analyse des enseignements tirés et l'identification des facteurs de réussite contribueront à une compréhension empirique plus approfondie des forces et des lacunes des réponses actuelles aux GAC, ce qui alimentera l'élaboration de pratiques et de politiques plus efficaces et appropriées.

\section{Approches en matière d'EGT relatives aux GAC}

En règle générale, les GAC remplissent simultanément des fonctions sécuritaires, politiques et économiques. Toutefois, trois grands types idéaux de GAC peuvent être identifiés. Selon la fonction principale qu'ils remplissent à un moment donné, ils peuvent être classés comme 1) des justiciers assurant la sécurité de leurs communautés ; 2) des milices travaillant sur l'injonction de commanditaires politiques ; ou 3) des gangs criminels poursuivant l'intérêt économique de leurs membres. Les approches relatives aux GAC peuvent être classées comme suivant trois logiques différentes : l'engagement, la gestion et la transformation ou EGT (engagement, management, and transformation ou EMT).

- L'engagement suit une logique à court terme ; les acteurs poursuivant cette approche le font pour des raisons instrumentales, c'est-à-dire parce qu'ils veulent assurer la sécurité de leurs propres membres du personnel lors de la mise en œuvre de leurs projets ou parce qu'ils veulent promouvoir la médiation et réduire la violence au sein des communautés.

- La gestion suit une logique à moyen terme car les acteurs envisagent un changement de fond dans les groupes ciblés. Les approches de gestion coercitive comprennent le recours à la force pour vaincre les GAC ou incarcérer leurs membres. Les approches de gestion coopérative s'efforcent de modifier les comportements et les rôles des GAC par la cooptation, la négociation ou la médiation.

- La transformation suit une logique à long terme et fait référence à un ensemble d'approches pour remplacer les fonctions que les GAC fournissent à leurs membres, commanditaires et aux communautés dans lesquelles ils sont installés au sein d'un État moderne et responsable lié par l'État de droit. La transformation s'attaque aux causes profondes et aux conditions structurelles qui ont conduit à l'émergence de l'écosystème des GAC. Cet objectif est difficile à atteindre car il nécessite de longs engagements et l'adhésion de multiples acteurs. 


\section{PRINCIPALES CONCLUSIONS}

\section{Indicateurs pour mesurer le succès des approches en matière d'EGT}

Compte tenu des multiples facteurs de confusion et des interventions qui se chevauchent, il est difficile de mesurer les résultats des approches en matière d'EGT, de les attribuer à des interventions spécifiques et d'évaluer leurs impacts. Ce rapport propose un ensemble d'indicateurs spécifiques que les acteurs intervenant et les tiers intéressés pourraient utiliser pour mesurer l'avancement des interventions en matière d'EGT dans la réalisation de leurs objectifs. Par exemple, un programme semble être sur la bonne voie pour atteindre une meilleure sécurité communautaire si les données montrent une augmentation du pourcentage de la population percevant une sécurité accrue et une diminution des incidents violents dans leurs communautés.

\section{Les GAC et la légitimité de l'État}

Les études existantes suggèrent que la fragilité de l'État est une cause clé de l'émergence des GAC. Cependant, le concept de fragilité étatique n'offre pas une explication universelle à la prolifération de tels groupes, notamment dans le cas d'États relativement forts caractérisés par un haut degré d'inégalités, y compris l'Afrique du Sud. Dans de tels contextes, ceux qui vivent dans les parties les plus aisées des principales villes bénéficient souvent ou tirent profit d'une protection opérationnelle assurée par la police et des entreprises de sécurité privées; cependant, ceux qui vivent dans des zones négligées se voient refuser l'accès à des systèmes de sécurité formels et se tournent donc vers les GAC, qui agissent comme des fournisseurs de sécurité informels. Par conséquent, il est important pour les acteurs intervenants de ne pas se concentrer exclusivement sur les efforts de construction de l'État, car ils pourraient ne pas s'attaquer aux causes profondes qui conduisent à l'émergence des GAC.

\section{De GAC à fournisseurs de sécurité communautaire?}

Si les justiciers se sont régulièrement transformés en milices politiques ou en criminels prédateurs, il existe aussi des exemples de GAC à forte fonction de sécurité qui ont évité une telle dévolution. Ce rapport identifie les facteurs de succès potentiels - y compris la présence de procédures de surveillance, d'un cadre juridique contraignant et de mécanismes de responsabilisation - que les acteurs intervenants peuvent prendre en compte lors de la conception de stratégies visant à modifier les caractéristiques internes et externes des GAC pour renforcer leur potentiel constructif et limiter leur potentiel destructeur. Ces facteurs de succès peuvent aider à transformer les GAC multidimensionnels en fournisseurs de sécurité communautaire plus responsables, capables et respectueux des règles.

\section{Le défi de la coordination des interventions en matière d'EGT}

La coordination des interventions en matière d'EGT peut être difficile en raison d'un manque de cohérence des politiques et parce que différents acteurs intervenants suivent des stratégies contradictoires à l'égard des GAC. En outre, différents acteurs intervenants, tels que les forces armées et les agences humanitaires, montrent des attitudes divergentes à l'égard de la coordination des approches en matière d'EGT. La cohérence et la coordination entre la multitude d'acteurs impliqués en matière d'EGT des GAC sont importantes pour le résultat global des interventions et pour la sécurité des acteurs intervenants 
et des communautés bénéficiaires. En outre, une meilleure coordination interorganisations peut aider à mettre en commun les ressources existantes et à les utiliser de manière plus efficace et durable en rationalisant les efforts et en diversifiant les sources de financement.

\section{INTRODUCTION}

La montée en puissance et le fonctionnement des groupes armés communautaires ou GAC (communitybased armed groups ou CBAGs) en Afrique subsaharienne font craindre qu'ils ne représentent un défi croissant pour les gouvernements et les citoyens de la région. ${ }^{1}$ Les communautés, les gouvernements, les organisations de la société civile, les agences humanitaires et de développement, ainsi que les fournisseurs de sécurité de ces environnements et d'autres encore ont développé une grande variété d'approches pour y faire face. Les GAC sont définis comme des groupes armés intégrés au sein des communautés et dont la délimitation peut être définie par le territoire, les liens du sang ou une identité partagée ; cette définition inclut les justiciers, les milices et les gangs criminels.

Comment les acteurs locaux, nationaux et internationaux ont-ils abordé le défi posé par les GAC et dans quelle mesure ces approches ont-elles été efficaces ? Cette étude vise à fournir un aperçu des conclusions pertinentes pour les politiques tirées de la vaste littérature sur les approches relatives aux GAC et à offrir des indicateurs pour évaluer le succès des initiatives achevées ou en cours en Afrique subsaharienne. La cartographie des approches, l'analyse des enseignements tirés et l'identification des facteurs de succès contribueront à une compréhension empirique plus approfondie des réponses à la prolifération des GAC, ce qui alimentera l'élaboration de pratiques et de politiques plus efficaces et appropriées. Les principales questions de recherche suivies par ce document de cartographie sont les suivantes:

- Quelles approches les États, la société civile et les acteurs internationaux ont-ils poursuivies pour engager, gérer et transformer les GAC en Afrique subsaharienne ?

- Quels enseignements et facteurs de réussite peuvent être identifiés à partir de la littérature sur les approches pour engager, gérer et transformer les GAC en Afrique subsaharienne?

- Quels indicateurs peuvent aider à mesurer le succès des approches pour engager, gérer et transformer les GAC en Afrique subsaharienne?

Le principal instrument de collecte de données utilisé était un examen détaillé de la littérature universitaire et grise sur les approches relatives aux GAC. En plus des articles universitaires, nous avons consulté des documents produits par des gouvernements, des organisations de la société civile, des groupes de réflexion et des agences internationales, y compris des termes de référence, des lignes directrices, des enseignements tirés ou des meilleures pratiques, des évaluations et des audits de projets, des fiches d'information et des rapports internes. Nous avons utilisé des recherches par mots-clés pour identifier les documents sources initiaux et nous avons ensuite utilisé l'échantillonnage en boule de neige en recherchant des citations à partir des sources initiales. La littérature universitaire et grise sur les sujets et approches couverts par ce document de cartographie était trop vaste pour être développée dans son intégralité. Cependant, un examen approfondi des publications les plus pertinentes par les principaux experts et institutions dans ce domaine a mis en évidence la littérature la plus pertinente.

1 Clionadh Raleigh, Pragmatisme et promiscuité : expliquer la montée de milices politiques compétitives en Afrique (Pragmatic and Promiscuous: Explaining the Rise of Competitive Political Militias across Africa), Journal of Conflict Resolution 60, $\mathrm{n}^{\circ} 2$ (2016) : 283-310. 


\section{Fonctions et types idéaux de GAC}

Contrairement à de nombreux groupes armés non étatiques ou GANE (non-state armed groups ou NSAGs), les GAC sont par définition intégrés dans leurs communautés, dont la délimitation peut être définie par le territoire, les liens du sang ou une identité partagée. Le concept de GAC exclut les prestataires de sécurité formels, telles que les entreprises privées de sécurité et les entreprises militaires. Les GANE à motivation politique comme les insurgés et les terroristes sont également exclus du concept de GAC parce que les GANE sont motivés idéologiquement ou religieusement et aspirent à prendre le contrôle de l'État pour établir un autre système politique. Les GAC ne poursuivent pas principalement une mission politique ; s'ils sont entraînés dans la sphère politique, ils agissent au nom d'entrepreneurs politiques dont les objectifs politiques sont de nature purement locaux. ${ }^{2}$

Bien que les GAC remplissent généralement plusieurs fonctions simultanément, trois types idéaux peuvent être discernés, selon leur fonction prédominante. Comme le montre le tableau 1, selon la fonction principale que les GAC remplissent à un moment donné, ils peuvent être classés comme des justiciers assurant la sécurité de leur communauté, comme des milices travaillant sur l'injonction de commanditaires politiques ou comme des gangs criminels poursuivant l'intérêt économique de leurs membres. ${ }^{3}$ Chaque type idéal peut être subdivisé en deux sous-types. Il est important de noter que les distinctions entre les différents types et sous-types idéaux de GAC peuvent être floues, et que les fonctions sont en constante évolution, selon des facteurs externes et de motivations internes.

\section{Tableau 1 : fonctions et types idéaux de GAC}

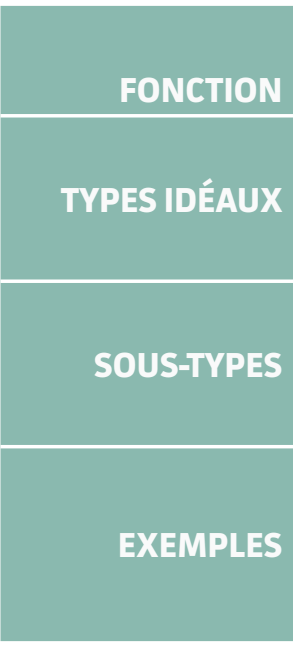

\section{SÉCURITÉ}

JUSTICIERS

GROUPES DE

LUTTE CONTRE

LA CRIMINALITÉ

BAKASSI BOYS

(NIGERIA)

\section{POLITIQUE}

MILICES

\begin{abstract}
MILICES
\end{abstract}
ETHNIQUES

D’AUTODÉFENSE

MAI MAI

ARMÉE BLANCHE

(RÉPUBLIQUE

DÉMOCRATIQUE

(SUD SOUDAN)

DU CONGO)
ÉCONOMIE/CRIMINALITÉ

GANGS

GANGS DE

JEUNES

CRIMINELS

POPULAIRES

ZANU PF YOUTH

MICROBES

GANGS À CAPE

(ZIMBABWE) (CÔTE D'IVOIRE)

FLATS

(AFRIQUE

DU SUD)

2 Moritz Schuberth, Le défi des groupes armées communautaires : vers une conceptualisation des milices, des gangs et des justiciers (The Challenge of Community-based Armed Groups: Towards a Conceptualization of Militias, Gangs, and Vigilantes), Contemporary Security Policy 36, $n^{\circ} 2$ (2015) : $296-320$. 
- Les justiciers peuvent être subdivisés en groupes de contrôle du crime dirigés en interne vers les membres de leurs propres communautés, ${ }^{4}$ comme les Bakassi Boys au Nigeria, ${ }^{5}$ et les groupes d'autodéfense protégeant les communautés contre les menaces externes, ${ }^{6}$ comme les Mai Mai et les Raia Mutomboki dans l'est de la République démocratique du Congo.7

- Les milices peuvent fonder leurs prétentions à la légitimité sur des notions d'ethnicité et d'identité proche ou clanique, comme l'Armée blanche de Nuer au Sud Soudan, ${ }^{8}$ ou sur une idéologie qui évoque l'image d'un ennemi commun des masses, comme les milices populaires composées de l'aile de jeunes du ZANU PF, le parti au pouvoir au Zimbabwe depuis l'indépendance. ${ }^{9}$

- Les gangs ont deux sous-types. Premièrement, les gangs de jeunes tels que les " microbes » de la capitale ivoirienne Abidjan qui sont de manière conventionnelle associés à la petite criminalité et à une sous-culture de la délinquance juvénile plutôt qu'à la criminalité organisée. ${ }^{10}$ Deuxièmement, les gangs criminels plus organisés et institutionnalisés, tels que les " Americans " ou les " Hard Livings » du Cap en Afrique du Sud, comprennent des membres de différentes cohortes d'âge et sont engagés dans des activités criminelles génératrices de profit, y compris le trafic de drogue. ${ }^{11}$

\section{REMISE EN CAUSE DES HYPOTHĖSES COMMUNES}

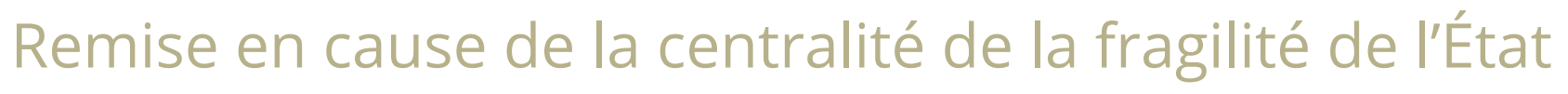

L'émergence des GAC est un thème commun de la littérature sur l'échec de l'État, sa faiblesse, son effondrement et sa fragilité. Les études de cette tradition situent généralement la prolifération des GAC dans le contexte de sociétés déchirées par la guerre, sujettes aux conflits ou post-conflits, où les responsabilités de base de l'État, telles que la fourniture de la sécurité, "tombent entre les mains de ceux qui se battront pour elle - seigneurs de guerre et chefs de gang $"{ }^{12}$ De ce point de vue, l'échec ou l'effondrement du système d'État westphalien depuis la fin de la guerre froide a entraîné la perte par

4 Les Johnston, Qu'est-ce que l'autodéfense ? (What is Vigilantism?), British Journal of Criminology 36, $\mathrm{n}^{\circ} 2$ (1996) : 232.

5 Kate Meagher, Détournement de la société civile : I'histoire vue de l'intérieur du groupe d'autodéfense les Bakassi Boys dans le sud-est du Nigeria (Hijacking Civil Society: The Inside Story of the Bakassi Boys Vigilante Group of South-eastern Nigeria), The Journal of Modern African Studies $45, \mathrm{n}^{\circ} 1$ (2007) : 89-115.

6 Raphael Garvin Abrahams. Citoyens justiciers : I'autodéfense et l'État (Vigilant Citizens: Vigilantism and the State), (Malden, Massachusetts : Polity Press, 1998) ; Bruce Baker, Fournisseurs non étatiques de services de sécurité dans les États africains fragiles (Nonstate Providers of Everyday Security in Fragile African States), dans Fragile States and Insecure People?, ed. Louise Andersen, Bjørn Møller, et Finn Stepputat (New York : Palgrave Macmillan, 2007), 123-47.

7 Kasper Hoffmann et Koen Vlassenroot, Les groupes armés et l'exercice du pouvoir public : les cas des Mayi-Mayi et des Raya Mutomboki à Kalehe, au Sud-Kivu (Armed Groups and the Exercise of Public Authority: The Cases of the Mayi-Mayi and Raya Mutomboki in Kalehe, South Kivu), Peacebuilding $2, n^{\circ} 2(2014): 202-20$.

8 John Young, Luttes populaires et cooptation de l'élite : I'Armée blanche de Nuer dans la guerre civile du Sud Soudan (Popular Struggles and Elite Co-optation: The Nuer White Army in South Sudan's Civil War), Genève : Small Arms Survey, 2016.

9 Norma Kriger, Les politiques du ZANU PF sous le gouvernement de « partage du pouvoir » au Zimbabwe (ZANU PF politics under Zimbabwe's ‘PowerSharing' Government), Journal of Contemporary African Studies 30, $n^{\circ} 1$ (2012) : 11-26.

10 Séverin Kouamé Yao, Nouchis, Ziguéhis et Microbes d'Abidjan : déclassement et distinction sociale par la violence de rue en Côte d'Ivoire, Politique Africaine $148, n^{\circ} 4$ (2017) : 89-107.

11 Théodore Petrus et Irvin Kinnes, Nouveaux bandits sociaux ? Une analyse comparative du gangstérisme dans les provinces de l'est et de l'ouest du Cap en Afrique du Sud (New Social Bandits? A Comparative Analysis of Gangsterism in the Western and Eastern Cape Provinces of South Africa), Criminology \& Criminal Justice 19, $\mathrm{n}^{\circ} 2$ (2018) : 179-96.

12 I. William Zartman, Introduction : poser le problème de l'effondrement de l'État (Introduction: Posing the Problem of State Collapse), dans Collapsed States: The Disin-tegration and Restoration of Legitimate Authority, éd. par I. William Zartman (Boulder, CO : Lynne Rienner, 1995), 1-11. 
l'État du monopole sur les moyens légitimes de violence. ${ }^{13}$ Cependant, un vivier émergent de chercheurs contestent le concept même d'échec de l'État comme étant conceptuellement défectueux, historiquement mal informé et culturellement biaisé ; ils soutiennent qu'il présente les démocraties occidentales libérales contemporaines comme le modèle universel, même pour les pays où un tel archétype n'a jamais existé et a peu de chances de s'enraciner. ${ }^{14}$

Les GAC sont également présents dans un certain nombre d'États relativement forts, viables et modernes. ${ }^{15}$ L'Afrique du Sud, par exemple, se classe dans la fourchette haute du milieu de l'indice des États fragiles ${ }^{16}$ et de l'indice de fragilité des États ${ }^{17}$, mais elle figure souvent parmi les dix pays les plus inégalitaires au monde. ${ }^{18}$ Dans un contexte d'États aussi forts mais inégaux, tous les citoyens ne sont pas affectés de la même manière par l'incapacité ou la réticence de l'État à assurer la sécurité. Ceux qui vivent dans les parties les plus aisées des principales villes bénéficient souvent ou tirent profit d'une protection opérationnelle assurée par la police et des entreprises de sécurité privées tandis que ceux qui vivent dans des zones négligées se voient refuser l'accès à des systèmes de sécurité formels et se tournent donc vers les GAC en tant que fournisseurs de sécurité informels. ${ }^{19}$ Dans les États à la fois fragiles et forts, les populations marginalisées vivant dans des zones à statut restreint se tournent vers des systèmes alternatifs de justice et de sécurité. ${ }^{20}$

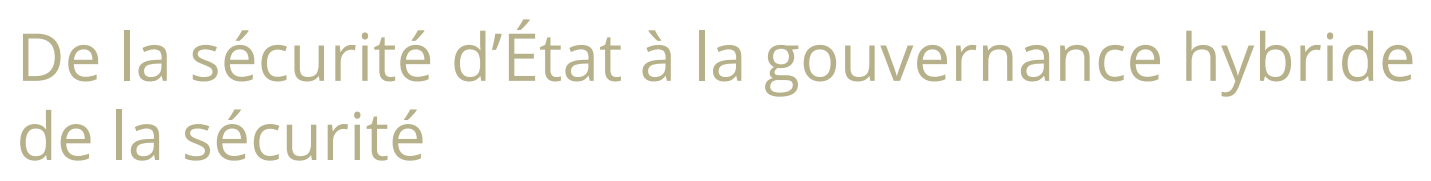

L'accent mis sur la fragilité de l'État se reflète également dans les notions traditionnelles de sécurité nationale ou de sécurité d'État qui considèrent la sécurité militaire comme conditionnelle à la capacité des gouvernements à repousser les menaces internes et externes à l'État-nation. Cependant, un nombre croissant de questions non traditionnelles ont également été érigées en menaces existentielles, telles que les flux migratoires ou le changement climatique. ${ }^{21}$ L'élargissement du cadre de sécurité à des objets de référence autres que la sécurité nationale ou d'État a entraîné un changement du paradigme de sécurité dominant. ${ }^{22}$ Ce changement a été

13 Edward Newman, État défaillant et ordre international : construire un monde post-wesphalien (Failed States and International Order: Constructing a Post-Westphalian World), Contemporary Security Policy 30, $n^{\circ} 3$ (2009) : 421-43 ; Max Weber, La politique comme vocation (Politics as a Vocation), dans From Max Weber: Essays in Sociology, eds. Hans Heinrich Gerth et C. Wright Mills (New York : Oxford University Press, 1946), 77-128.

14 Charles T. Call, La fausse hypothèse de l' "État défaillant » (The Fallacy of the 'Failed State'), Third World Quarterly 29, n 8 (2008) : 1491-1507; Mark Duffield, Développement, sécurité et guerre sans fin : gouverner le monde des populations (Development, Security and Unending War: Governing the World of Peoples), Cambridge : Polity Press, 2007).

15 Moritz Schuberth, Remettre en cause l'hypothèse des États faibles: I'autodéfense en Afrique du Sud et au Brésil (Challenging the Weak States Hypothesis: Vigilantism in South Africa and Brazil), Journal of Peace, Conflict \& Development 20 (2013) : 38-51.

16 L'Afrique du Sud se classe 86e sur 178 pays. Voir l'indice 2018 des États fragiles du Fonds pour la paix. http://fundforpeace.org/fsi/.

17 L'Afrique du Sud atteint 8 points de fragilité sur 25. Voir Monty G. Marshall et Gabrielle C. Elzinga-Marshall, Rapport mondial 2017 : conflit, gouvernance et fragilité de l'État (Global Report 2017: Conflict, Governance and State Fragility) Vienne, VA : Center for Systemic Peace, 2017$), 48$.

18 Voir l'indice GINI de la Banque mondiale. https://data.worldbank.org/indicator/SI.POV.GINI?page. L'indice GINI examine la répartition des revenus ou de la richesse d'un pays, où 0 représente l'égalité totale et 100 l'inégalité totale. L'Afrique du Sud présente un coefficient de Gini élevé de 63.

19 David Pratten et Atreyee Sen, Justiciers mondiaux : perspectives sur la justice et la violence (Global Vigilantes: Perspectives on Justice and Violence) (Oxford : Oxford University Press, 2007).

20 Thomas Risse, Gouvernance dans des domaines de statut limité de l'État : introduction et aperçu (Governance in Areas of Limited Statehood: Introduction and Overview), dans Governance without a State? Policies and Politics in Areas of Limited State Hood, ed. Thomas Risse (New York: Columbia University Press, 2011), 1-35.

21 Nana Poku et David T. Graham, eds. Redéfinir la sécurité : mouvements de populations et sécurité nationale (Redefining Security: Population Movements and National Security), Westport, CT : Greenwood Publishing Group, 1998); Oli Brown, Anne Hammill et Robert McLeman, Le changement climatique en tant que nouvelle menace à la sécurité : implications pour l'Afrique (Climate Change as the 'New' Security Threat: Implications for Africa), International Affairs 83, nº 6 (2007) : 1141-54.

22 Barry Buzan, Ole Wæver et Jaap De Wilde, Sécurité : un nouveau cadre pour l'analyse (Security: A New Framework for Analysis), Boulder, CO : Lynne Rienner Publishers, 1998. 
particulièrement bien perçu dans le concept de sécurité humaine, qui comprend à la fois la notion d'être "libéré de la terreur " et d'être "libéré de la misère ». ${ }^{23}$ Cependant, la définition globale de la sécurité humaine, y compris les droits et libertés positifs et négatifs, a été critiquée comme trop vague, ce qui rend difficile pour les décideurs politiques de donner la priorité à certaines menaces par rapport à d'autres. ${ }^{24}$

Dans le contexte des GAC, le concept de sécurité communautaire pourrait être plus utile car il comble le fossé entre les initiatives axées sur le niveau de l'État et celles axées sur l'individu comme point de départ. ${ }^{25}$ La sécurité communautaire peut être conceptualisée à la fois comme état final et comme processus menant à cet état final. Lorsqu'elle est considérée comme un état final, la sécurité communautaire « est la situation dans laquelle les communautés se sentent en sécurité face aux menaces exercées par des conflits violents [...], la criminalité et un manque de protection [...] de la part de l'État $॥ .{ }^{26}$ Lorsqu'elle est comprise comme un processus, la sécurité communautaire « signifie que les communautés participent à l'identification et à la hiérarchisation de leurs besoins en matière de sécurité, ainsi qu'au développement et à la mise en œuvre de réponses appropriées à leurs besoins en matière de sécurité. » En d'autres termes, les membres de la communauté sont à la fois les bénéficiaires et les agents de la sécurité humaine. Dans cette perspective, l'amélioration de la sécurité communautaire en tant qu'état final peut être considérée comme l'objectif global des approches en matière d'EGT des GAC ; les approches en matière d'EGT peuvent être considérées comme sécurité communautaire dans sa conception en tant que processus. ${ }^{27}$

\section{De fournisseurs de sécurité à sources d'insécurité}

Suite aux critiques justifiées des États défaillants et des paradigmes de sécurité nationale, l'attention s'est déplacée vers des dispositifs de sécurité communautaire qui assurent la sécurité " d'en bas " ou " du point de vue des utilisateurs finaux $"{ }^{28}$ Bien que reconnaissant que ces dispositifs hybrides de sécurité comprennent des milices et des groupes de crime organisé, leurs défenseurs soulignent la fourniture relativement efficace de la sécurité par les GAC, par rapport aux acteurs de la sécurité de l'État et à la légitimité locale plus élevée dont jouissent ces groupes. ${ }^{29}$ De ce point de vue, les GAC pourraient offrir la meilleure option disponible pour une fourniture efficace de la justice et de la sécurité à court terme. ${ }^{30} \mathrm{En}$ même temps, les GAC pourraient être considérés comme plus légitimes en raison de leurs liens étroits avec les pratiques culturelles locales et de leur plus grand alignement avec les attitudes et normes

23 PNUD, Rapport sur le développement humain de 1994 (Human Development Report 1994), New York: Programme des Nations Unies pour le développement, 1994), 22.

24 Edward Newman, Sécurité humaine et constructivisme (Human Security and Constructivism), International Studies Perspectives 2, $\mathrm{n}^{\circ} 3$ (2001) : 243.

25 Andrew McLean, Sécurité communautaire et cohésion sociale : vers une approche pour le PNUD (Community Security and Social Cohesion: Towards a UNDP Approach), Genève : Programme des Nations Unies pour le développement, 2009.

26 Willemijn Verkoren, Rens Willems, Jesper Kleingeld et Hans Rouw, De DDR à la promotion de la sécurité : relier les programmes nationaux aux initiatives communautaires (From DDR to Security Promotion: Connecting National Programs to Community Initiatives), International Journal of Peace Studies 15, $n^{\circ} 2$ (2010) : 4 .

27 Will Bennett, Manuel de la sécurité communautaire (Community Security Handbook), Londres : Saferworld, 2014.

28 Alexandra Abello Colak et Jenny Pearce, Sécurité « d'en bas » dans le contexte de la violence chronique ('Security from Below' in Contexts of Chronic Violence), IDS Bulletin 40, $n^{\circ} 2$ (2009) : 11-19; Robin Luckham et Tom Kirk, Les deux faces de la sécurité dans les commandes politiques hybrides : un cadre pour l'analyse et la recherche (The Two Faces of Security in Hybrid Political Orders: A Framework for Analysis and Research), Stability: International Journal of Security \& Development 2, $\mathrm{n}^{\circ} 2$ (2013).

29 Volker Boege, M. Anne Brown et Kevin P. Clements, Des commandes politiques hybrides, pas des États fragiles (Hybrid Political Orders, not Fragile States), Peace Review 21, n 1 (2009) : 13-21.

30 Bruce Baker et Eric Scheye, Fourniture de justice et de sécurité multi-niveaux dans les États post-conflits et fragiles : une analyse (Multi-Layered Justice and Security Delivery in Post-Conflict and Fragile States: Analysis), Conflict, Security \& Development 7, $n^{\circ} 4$ (2007) : 503-28. 
sociales de leurs communautés. ${ }^{31}$ En l'absence de mécanismes de supervision et de responsabilité solides, cependant, les GAC pourraient se transformer, passant de fournisseurs de sécurité à sources d'insécurité.

La littérature sur l'autodéfense dépeint un changement général de motivation qui transforme les justiciers consciencieux assurant la sécurité de leurs communautés en bandes criminelles intéressées ou en milices soutenues par l'État. ${ }^{32}$ Dans le contexte de l'Afrique subsaharienne, des études ont mis en garde que «la plupart du temps, ils se transforment en criminels, outre le mandat que leur a donné la communauté de monter la garde dans le quartier ${ }^{33}$ et qu'au Kenya, entre autres, les [j]usticiers peuvent se transformer en gangs, et les gangs peuvent se transformer en milices armées $"{ }^{34}$ Cependant, ce document identifie des cas de GAC, y compris les Arrow Boys en Ouganda, qui ne se sont pas transformés en gangs ou milices prédatrices en raison de facteurs externes, tels qu'un contrôle étatique fort ou un cadre juridique favorable. La transformation des justiciers en sources d'insécurité communautaire n'est donc pas l'inévitable trajectoire des GAC.

\section{Outils standard comme panacée universelle}

Au cours des dernières décennies, de nombreux acteurs nationaux et internationaux ont développé un ensemble d'outils standard pour engager, gérer et transformer les GAC. ${ }^{35}$ L'utilisation de ces outils " universels » présente des avantages pratiques, mais elle peut également être inadaptée lorsqu'elle est appliquée à de nouveaux environnements et dans différentes circonstances. ${ }^{36} \mathrm{D}^{\prime i m p o r t a n t e s}$ différences existent, par exemple, entre les groupes armés urbains et ruraux, ou entre les GAC qui ont émergé en réponse aux petits criminels par rapport à ceux qui se sont formés pour lutter contre les groupes extrémistes violents. ${ }^{37}$ Pourtant, les acteurs nationaux et internationaux ont tardé à s'adapter au nouvel environnement opérationnel des villes où les GAC sont présents, comme Nairobi, au Kenya, ou Beni, dans l'est de la République démocratique du Congo. ${ }^{38}$

Dans la capitale haïtienne, Port-au-Prince, une Mission des Nations Unies pour la stabilisation en Haïti (MINUSTAH) a tenté de traiter les GAC urbains de la même manière que les rebelles ruraux en Afrique centrale. En raison d'une mauvaise compréhension du contexte et de la nature des GAC en Haïti, la MINUSTAH a été initialement chargée de mettre en œuvre un programme traditionnel de désarmement, de démobilisation et de réintégration (DDR) malgré le fait que l'environnement conflictuel n'était pas propice à un tel effort. Lorsque l'échec de cette stratégie initiale est devenu clair, la MINUSTAH a modifié son approche pour adopter un programme plus adapté de réduction de la violence communautaire ou

31 Lisa Denney, Surmonter la division État/non-État : une approche utilisateur final de la réforme de la sécurité et de la justice (Overcoming the State/ Non-State Divide: An End User Approach to Security and Justice Reform), International Peacekeeping 21, n 2 (2014) : 254.

32 H. Jon Rosenbaum et Peter C. Sederberg, L'autodéfense : une analyse de l'établissement de la violence (Vigilantism: an analysis of establishment violence), Comparative Politics 6, $n^{\circ} 4$ (1974) : 541-570; Abrahams, justiciersVigilant Citizens, 22.

33 Gani Joses Yoroms, Les milices en tant que phénomène social : vers une construction théorique (Militias as a social phenomenon: Towards a theoretical construction), dans Civil Militia: Africa's Intractable Security Menace?, ed. David J. Francis (New York : Routledge, 2005), 40.

34 Mikewa Ogada et Njonjo Mue, Réforme du secteur de la sécurité et justice transitionnelle au Kenya (Security Sector Reform and Transitional Justice in Kenya), Nairobi : International Center for Transitional Justice, 2010: 3.

35 Robert Muggah et Chris O’Donnell, Désarmement, démobilisation et réintégration de prochaine génération (Next Generation Disarmament, Demobilization and Reintegration), Stability: International Journal of Security \& Development $4, \mathrm{n}^{\circ} 1$ (2015).

36 Michael N. Barnett et Martha Finnemore, Les politiciens, le pouvoir et les pathologies des organisations internationales (The Politics, Power, and Pathologies of International Organizations), International Organization 53, $\mathrm{n}^{\circ} 4$ (1999) : 699-732.

37 Stina Torjesen, Nouvelles avenues pour la recherche et l'étude de DDR (New Avenues for Research in the Study of DDR), Conflict, Security \& Development $9, n^{\circ} 4$ (2009) : 411.

38 Marion Harroff-Tavel, Violence et action humanitaire dans les zones urbaines : nouveaux défis, nouvelles approches (Violence and Humanitarian Action in Urban Areas: New Challenges, New Approaches), International Review of the Red Cross 92, n 878 (2010) : 329-50. 
RVC (Community Violence Reduction ou CVR). Le programme de RVC visait à dissuader des sections à risque de la population de rejoindre les GAC d'Haïti, qui ont plus en commun avec les gangs de rue qu'avec les forces rebelles rurales - les cibles conventionnelles du DDR. ${ }^{39}$ Même si le Département des opérations de maintien de la paix de l'ONU considérait les activités de RVC comme un modèle pour les efforts de DDR dans les missions de stabilisation de I'ONU en République démocratique du Congo, au Mali et au Sud-Soudan, d'autres participants se demandaient comment une approche adaptée aux gangs urbains pouvait être personnalisée pour les groupes armés ruraux. ${ }^{40}$

\section{CARTOGRAPHIE DES APPROCHES EN MATIÈRE D’EGT DES GAC EN AFRIQUE SUB SAHARIENNE}

\section{Logique sous-jacente aux approches relatives aux GAC}

Au cours de la dernière décennie, les cercles politiques ont de plus en plus débattu de la manière dont les agences internationales travaillant dans les pays sortant d'un conflit devraient traiter les GANE, ${ }^{41}$ en particulier dans les États fragiles et touchés par un conflit. ${ }^{42}$ Par exemple, Stedman a compté trois stratégies utilisées par les acteurs intervenant vis-à-vis des groupes "saboteurs " - des groupes qui utilisent la violence pour saper la paix dans leur propre intérêt. Les trois stratégies sont l'incitation, la coercition et la socialisation. L'incitation implique de "donner au saboteur ce qu'il veut", et les stratégies coercitives reposent sur « l'utilisation ou la menace de sanctions pour dissuader ou altérer un comportement inacceptable du saboteur $" .^{43}$ La socialisation combine des carottes et des bâtons en construisant des normes et des valeurs qui définissent quel comportement est acceptable et quelles exigences sont légitimes, modifiant ainsi l'écosystème dans lequel les saboteurs opèrent. ${ }^{44}$

Bien que le concept de saboteurs ait initialement été limité aux contextes dans lesquels un accord de paix entre deux factions belligérantes avait été signé, il a depuis été appliqué de manière plus libérale à une série de GANE ${ }^{45}$ et même à des groupes du crime organisé. ${ }^{46}$ À titre d'exemple, Hofmann et Schneckener ont classé les stratégies en faveur des GANE comme étant la négociation, la force/l'effet de levier et la persuasion, ce qui

39 Moritz Schuberth, La transformation de la violence politique à la violence criminelle ? (A Transformation from Political to Criminal Violence?), Politique, crime organisé et fonctions changeantes dans les groupes urbains armés d'Haïti (Politics, Organised Crime and the Shifting Functions of Haiti's Urban Armed Groups), Conflict, Security \& Development 15, $n^{\circ} 2$ (2015) : 169-96.

40 Moritz Schuberth, Désarmement, démobilisation et réintégration dans des contextes non conventionnels : le cas de la réduction de la violence de la MINUSTAH (Disarmament, Demobilisation and Reintegration in Unconventional Settings: The Case of MINUSTAH's Community Violence Reduction), International Peacekeeping 24, $\mathrm{n}^{\circ} 3$ (2017) : 427 ; Hugo De Vries, Les fluctuations de la stabilisation au Congo (The Ebb and Flow of Stabilization in the Congo), Londres/Nairobi : Rift Valley Institute, 2016) : 3.

41 Claudia Hofmann et Ulrich Schneckener, ONG et acteurs armés non étatiques : améliorer la conformité avec les normes internationales (ONG and Nonstate Armed Actors: Improving Compliance with International Norms) Washington, DC : United States Institute of Peace, 2011).

42 Sukanya Podder, De saboteurs à bâtisseurs de l'État : des approches constructives pour l'engagement avec les groupes armés non étatiques dans les États fragiles (From Spoilers to Statebuilders: Constructive Approaches to Engagement with Non-state Armed Groups in Fragile States), Paris : OCDE Publishing, 2012).

43 Stephen John Stedman, Problèmes de saboteurs dans les processus de paix (Spoiler Problems in Peace Processes), International Security $22, \mathrm{n}^{\circ} 2$ (1997) : 13.

44 Ibid.

45 Matthew Hoddie et Caroline A. Hartzell, Renforcer la paix dans les États après la guerre civile : transformer les saboteurs en parties prenantes (Strengthening Peace in Post-Civil War States: Transforming Spoilers into Stakeholders), Chicago et Londres : University of Chicago Press, 2010.

46 Mark Shaw et Walter Kemp, Repérer les saboteurs : un guide pour analyser le crime organisé (Spotting the Spoilers: A Guide to Analyzing Organized Crime), New York : International Peace Institute, 2012). 
peut être considéré comme correspondant à l'incitation, la coercition et la socialisation de Stedman, comme indiqué dans le tableau $2 .{ }^{47}$ Cependant, contrairement à la typologie de Stedman, Hofmann et Schneckener suggèrent que la force/l'effet de levier " implique un mélange de bâtons et de carottes ", classant ainsi les pots-de-vin et les discussions avec des éléments modérés au sein des GANE comme coercitifs. ${ }^{48}$

\section{Tableau 2 : échéanciers, objectifs et exemples d'approches en matière d'EGT}

\begin{tabular}{|c|c|c|c|}
\hline APPROCHE & ENGAGEMENT & GESTION & TRANSFORMATION \\
\hline ÉCHÉANCIERS & COURT TERME & MOYEN TERME & LONG TERME \\
\hline OBJECTIFS & $\begin{array}{r}\text { ACCÈS SÉCURISÉ ; AMÉLIORATION } \\
\text { DES RELATIONS ENTRE LES } \\
\text { COMMUNAUTÉS ET LES GAC }\end{array}$ & $\begin{array}{l}\text { CHANGEMENT DE RÔLES, DE PORTÉE } \\
\text { ET DE COMPORTEMENTS DES GAC }\end{array}$ & $\begin{array}{r}\text { MODIFICATION DES CAUSES } \\
\text { PROFONDES ET DES } \\
\text { CONDITIONS STRUCTURELLES } \\
\text { DE L'ÉCOSYSTÈME DES GAC }\end{array}$ \\
\hline EXEMPLES & $\begin{array}{r}\text { COMMUNAUTÉS DE BARAZA ET } \\
\text { NÉGOCIATIONS D'ACCÈS PAR LE CICR } \\
\text { ET MSF DANS L'EST DE LA RÉPUBLIQUE } \\
\text { DÉMOCRATIQUE DU CONGO }\end{array}$ & $\begin{array}{l}\text { DISCUSSIONS AVEC LES } \\
\text { GRANDS TRAFIQUANTS AU } \\
\text { MALI; COOPTATION DE LA } \\
\text { FICC PAR L'ÉTAT NIGÉRIAN }\end{array}$ & $\begin{array}{r}\text { DDR, RSS ET RVC DANS LES MISSIONS } \\
\text { DE STABILISATION DE L'ONU EN } \\
\text { RÉPUBLIQUE CENTRAFRICAINE, EN } \\
\text { RÉPUBLIQUE DÉMOCRATIQUE DU } \\
\text { CONGO ET AU MALI }\end{array}$ \\
\hline $\begin{array}{r}\text { LITTÉRATURE } \\
\text { SUR LES } \\
\text { SABOTEURS } \\
\text { (STEDMAN, 1997) }\end{array}$ & INCITATION & COERCITION & SOCIALISATION \\
\hline $\begin{array}{l}\text { LITTÉRATURE } \\
\text { SUR LES GANE } \\
\text { (SCHNECKENER, 2009) }\end{array}$ & MARCHANDAGE & FORCE/EFFET DE LEVIER & PERSUASION \\
\hline
\end{tabular}

Source : auteur.

II peut sembler logique d'inclure des aspects à la fois coopératifs et coercitifs sous la bannière force/effet de levier, mais nous proposons d'utiliser le terme global de gestion pour ces deux approches afin de modifier le comportement des GAC. Nous utilisons l'engagement plutôt que la négociation ou l'incitation pour les approches qui ne tentent pas de modifier le comportement des GAC, et la transformation plutôt que la socialisation ou la persuasion pour les approches qui tentent de modifier les conditions structurelles de l'écosystème des GAC. La terminologie de l'engagement, de la gestion et de la transformation ou EGT

47 Ulrich Schneckener, Saboteurs ou acteurs de la gouvernance ? Engager les groupes armés non étatiques dans les zones de statut limité de l'État (Spoilers or Governance Actors? Engaging Armed Non-State Groups in Areas of Limited State Hood), SFB- Governance Working Paper Series, $n^{\circ} 21$ (Berlin : DFG Research Center (SFB) 700, 2009.

48 Claudia Hofmann et Ulrich Schneckener, Engager les acteurs armés non étatiques dans la construction de l'État et de la paix : options et stratégies (Engaging Non-State Armed Actors in State- and Peace-Building: Options and Strategies), International Review of the Red Cross $93, \mathrm{n}^{\circ} 883$ (2011) : 610. 
(engagement, management, and transformation ou EMT) reconnaît le spectre des réponses disponibles aux GAC au-delà des outils standard tels que le DDR et la RSS, ainsi que les différents échéanciers pendant lesquels les acteurs intervenants peuvent raisonnablement être censés concentrer leurs efforts en matière d'EGT sur des GAC spécifiques. Ces échéanciers vont de l'engagement à court terme à la transformation à long terme.

\section{Engagement}

L'engagement suit une logique à court terme ; les acteurs poursuivant cette approche le font pour des raisons instrumentales parce qu'ils veulent assurer la sécurité de leurs propres membres du personnel lors de la mise en œuvre de leurs projets ou parce qu'ils veulent promouvoir la médiation entre les GAC et leurs communautés respectives. L'engagement fait référence, par exemple, aux systèmes traditionnels de résolution des conflits communautaires, tels que les communautés de barazas dans l'est de la République démocratique du Congo. ${ }^{49}$ Un autre exemple d'engagement est fourni par les acteurs de l'humanitaire et du développement, tels que le Comité international de la Croix-Rouge (CICR) ou Médecins sans Frontières (MSF) ; ces groupes mettent en œuvre des projets dans les zones contrôlées par des GAC en République démocratique du Congo et doivent engager les membres de ces groupes pour obtenir un accès sûr pour leurs membres du personnel. ${ }^{50}$ Bien que cette approche ne cherche pas à modifier le comportement ou l'environnement structurel des GAC, elle peut par inadvertance renforcer leur autonomie vis-à-vis de l'État ou des communautés dans lesquelles ils sont basés si les acteurs intervenants fournissent une compensation financière ou en nature en échange d'un accès sûr.

\section{Gestion}

Les approches en matière de gestion suivent une logique à moyen terme, prévoyant un changement substantiel des groupes ciblés ; elles visent à avoir un impact direct sur le rôle, la portée et le comportement des GAC, par des moyens coercitifs ou coopératifs.

Les approches de gestion coercitive impliquent les forces de sécurité et incluent l'utilisation de la force pour vaincre les GAC ou incarcérer leurs membres, comme des descentes de police militarisées contre des gangs en Afrique du Sud. ${ }^{51} \mathrm{Si}$ des approches coercitives ciblent sans discernement les communautés dans lesquelles les GAC sont basées, elles peuvent involontairement affaiblir la légitimité de l'État et renforcer les liens entre les GAC et leurs communautés, comme ce fut le cas pour les gangs organisés au Cap. ${ }^{52}$

Les approches de gestion coopérative s'efforcent de modifier les comportements et les rôles des GAC par le biais de la cooptation, de la négociation ou de la médiation. Au Mali, par exemple, les dirigeants locaux impliqués dans les économies illicites (grands trafiquants) ont été invités à participer aux

49 Phil Clark, Ethnicité, leadership et médiation de conflit dans l'est de la République démocratique du Congo : le cas des Barza intercommunautaires (Ethnicity, Leadership and Conflict Mediation in Eastern Democratic Republic of Congo: The Case of the Barza Inter-Communautaire), Journal of Eastern African Studies 2, $\mathrm{n}^{\circ} 1$ (2008) : 8 .

50 Johan Pottier, L'ethnographie comme barrière : négocier l'accès humanitaire en Ituri, dans l'est de la RDC (Roadblock Ethnography: Negotiating Humanitarian Access in Ituri, Eastern DR Congo), 1999-2004, Africa: Journal of the International African Institute 76, $\mathrm{n}^{\circ} 2$ (2006) : 151-79.

51 Guy Lamb, Militarisation de la police et la guerre contre la criminalité en Afrique du Sud (Police Militarisation and the 'War on Crime' in South Africa), Journal of Southern African Studies 44, $n^{\circ} 5$ (2018) : 933-49.

52 Petrus et Kinnes, Nouveaux bandits sociaux ? (New Social Bandits?), 189. 
négociations entre le gouvernement et les groupes rebelles touaregs. ${ }^{53}$ En République centrafricaine, la médiation locale entre les anciens rebelles Séléka et les groupes d'autodéfense anti-balaka a été plus fructueuse que les efforts de médiation internationale et régionale. ${ }^{54}$ La négociation et la médiation peuvent améliorer le comportement positif des GAC, mais elle peut aussi renforcer leur légitimité. La cooptation, en revanche, peut renforcer la légitimité de l'État et des GAC; elle peut aussi conduire au déracinement des GAC et à des comportements plus abusifs vis-à-vis de leurs communautés, comme cela s'est produit avec la Force d'intervention civile conjointe ou FICC (Civilian Joint Task Force ou CJTF) à la suite de la cooptation de l'État nigérian. ${ }^{55}$

\section{Transformation}

La transformation désigne un ensemble d'approches visant à remplacer les fonctions que les GAC remplissent pour leurs membres, leurs commanditaires et les communautés dans lesquelles ils sont installés au sein d'un État moderne et responsable lié par l'État de droit. Selon la logique de transformation, cela peut être réalisé en brisant le patronage entre les GAC et leurs commanditaires politico-criminels par la démobilisation, le désengagement et la réintégration (DDR), en renforçant les forces de sécurité de l'État par une réforme du secteur de la sécurité (RSS) et en utilisant la réduction de la violence communautaire (RVC) pour couper les liens entre les GAC et leurs communautés. À titre d'exemple, les missions de stabilisation de l'ONU en République centrafricaine, en République démocratique du Congo et au Mali ont toutes utilisé une combinaison de DDR, de RSS et de RVC, avec des degrés variables de succès. ${ }^{56}$ Dans une logique à long terme, la transformation vise à répondre aux causes profondes et aux conditions structurelles qui ont conduit à l'émergence de l'écosystème des GAC. Cette approche nécessite de longs délais d'engagement et l'adhésion d’acteurs locaux, nationaux et internationaux.

En croisant le cadre de l'EGT avec les trois fonctions des GAC, nous avons développé une matrice d'intervention d'approches standards des GAC. Comme le montre le tableau 3, la matrice d'intervention répertorie les outils standards habituellement utilisés pour gérer les GAC. La matrice est issue de la conceptualisation théorique basée sur une revue approfondie de la littérature. Il est possible et important de coordonner des stratégies selon l'axe des ordonnées pour s'assurer que l'approche E, M ou T traite les trois fonctions des GAC par exemple en combinant la RSS, le DDR et la RVC. En revanche, la coordination selon l'axe des abscisses, par exemple entre les approches coercitives et coopératives pour gérer les GAC, est difficile parce que les différentes approches suivent des logiques institutionnelles contradictoires.

53 Katrin Planta et Véronique Dudouet, Prêts à négocier ? Options et risques de la transformation politique des groupes armés non conventionnels (Fit for Negotiation? Options and Risks in the Political Transformation of Non-conventional Armed Groups). Oslo : Norwegian Peacebuilding Resource Centre (NOREF), 2015, 5.

54 Marie-Joëlle Zahar et Delphine Mechoulan, La paix un élément à la fois ? La médiation locale et la paix durable en République centrafricaine (Peace by Pieces? Local Mediation and Sustainable Peace in the Central African Republic) (New York : International Peace Institute, 2017).

55 Kyle Dietrich, Quand on ne voit pas l'ennemi, les civils deviennent l'ennemi : vivre pendant les six ans d'insurrection au Nigeria (When We Can't See the Enemy, Civilians Become the Enemy: Living Through Nigeria's Six-Year Insurgency) Washington, DC : Center for Civilians in Conflict, 2015.

56 Arthur Boutellis, L'ONU peut-elle stabiliser le Mali ? Vers une doctrine de stabilisation de I'ONU (Can the UN Stabilize Mali? Towards a UN Stabilization Doctrine?), Stability: International Journal of Security and Development 4, $\mathrm{n}^{\circ} 1$ (2015); Muggah et O’Donnell, Désarmement, démobilisation et réintégration de prochaine génération (Next Generation Disarmament, Demobilization and Reintegration); De Vries, Les fluctuations de la stabilisation au Congo (The Ebb and Flow of Stabilization in the Congo). 
Tableau 3 : Outils standards utilisés par les acteurs intervenant pour gérer les GAC

\begin{tabular}{|c|c|c|c|c|c|}
\hline & \multicolumn{4}{|c|}{ APPROCHES RELATIVES AUX GAC } \\
\hline & & \multirow{2}{*}{ ENGAGEMENT } & \multicolumn{2}{|c|}{ GESTION } & \multirow{2}{*}{ TRANSFORMATION } \\
\hline & & & COERCITIVE & COOPÉRATIVE & \\
\hline \multirow{3}{*}{ 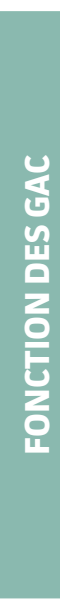 } & SÉCURITÉ & $\begin{array}{c}\text { ENGAGER DES GAC } \\
\text { COMME PRESTATAIRES } \\
\text { DE SÉCURITÉ }\end{array}$ & $\begin{array}{l}\text { VAINCRE LES GAC } \\
\text { (PACIFICATION) }\end{array}$ & $\begin{array}{l}\text { COOPTATION DES GAC } \\
\text { (GOUVERNANCE DE } \\
\text { LA SÉCURITÉ } \\
\text { HYBRIDE) }\end{array}$ & $\begin{array}{l}\text { REMPLACER LES GAC } \\
\text { PAR LA POLICE } \\
\text { (RSS) }\end{array}$ \\
\hline & POLITIQUES & $\begin{array}{l}\text { ENTRER DANS LES } \\
\text { ZONES CONTRÔLÉES } \\
\text { PAR LES GAC } \\
\text { AVEC DES OSC }\end{array}$ & $\begin{array}{l}\text { OBTENIR UN SOUTIEN } \\
\text { LOCAL } \\
\text { (CONTRE- } \\
\text { INSURRECTION) }\end{array}$ & $\begin{array}{l}\text { NÉGOCIER AVEC LES GAC } \\
\text { (PARTAGE DU POUVOIR) }\end{array}$ & $\begin{array}{l}\text { REMPLACER LE RÉSEAU } \\
\text { DE COMMANDITAIRES } \\
\text { (DDR) }\end{array}$ \\
\hline & $\begin{array}{l}\text { ÉCONOMIE/ } \\
\text { CRIMINALITÉ }\end{array}$ & $\begin{array}{l}\text { GAC SECONDAIRES } \\
\text { DANS LES } \\
\text { PLATEFORMES } \\
\text { COMMUNAUTAIRES }\end{array}$ & $\begin{array}{l}\text { MEMBRES DES GAC } \\
\text { INCARCÉRÉS } \\
\text { (GUERRE CONTRE } \\
\text { LES GANGS) }\end{array}$ & $\begin{array}{l}\text { MÉDIATION ENTRE LES GAC } \\
\text { (TRÊVE DES GANGS) }\end{array}$ & $\begin{array}{l}\text { RÉDUIRE L'APPEL } \\
\text { À REJOINDRE LES GAC } \\
\text { (RVC) }\end{array}$ \\
\hline & & & & & Source: auteur. \\
\hline
\end{tabular}

Parmi les résultats escomptés des approches en matière d'EGT figurent un comportement protecteur plutôt que prédateur vis-à-vis des communautés locales; une approche durable ; l'identification de toutes les parties prenantes clés et la coordination entre les partenaires de mise en œuvre; le respect des principes de sensibilité aux conflits; et l'adhésion des GAC aux normes et aux normes de bonne gouvernance, telles que les droits de l'Homme et l'État de droit. Compte tenu des multiples facteurs de confusion et des interventions qui se chevauchent, il est difficile de mesurer les résultats des approches, de les attribuer à des interventions spécifiques et d'évaluer leurs impacts. ${ }^{57}$ Le tableau 4 fournit un exemple d'indicateurs potentiels que les acteurs intervenants et les tiers intéressés pourraient utiliser pour mesurer l'impact des interventions en matière d'EGT.

Bien que l'objectif global des approches en matière d'EGT soit une amélioration de la sécurité communautaire des populations vivant dans les zones où des GAC sont présents, chaque approche EGT a son objectif spécifique. L'engagement se concentre sur la garantie d'un accès sûr des acteurs intervenant dans les zones où des GAC sont présents ; la gestion coercitive vise à réduire la portée et la légitimité des GAC; la gestion coopérative s'efforce d'améliorer le traitement des populations locales par les GAC ; et la transformation aspire à rendre les GAC obsolètes pour leurs membres, commanditaires et communautés. Le tableau 4 propose deux indicateurs spécifiques, chacun pour mesurer les progrès dans l'atteinte du but global et des quatre objectifs. Par exemple, un programme semble être sur la bonne voie pour atteindre une meilleure sécurité communautaire si les données montrent une augmentation

57 Tariro Mutongwizo, Lezanne Leoschut et Patrick Burton, Copropriété et collaboration : réflexions sur la mesure des impacts et des changements basées sur les partenariats de prévention de la violence entre les communautés et l'État sur la base de données (Co-ownership and Collaboration: Insights into the Measurement of Impact and Change from Evidence-based Community and State Violence Prevention Partnerships), Stability: International Journal of Security \& Development $4, n^{\circ} 1$ (2015). 
du pourcentage de la population percevant une sécurité accrue et une diminution des incidents violents signalés dans leurs communautés.

\section{Tableau 4 : Indicateurs pour mesurer les résultats des différentes approches des GAC}

\author{
RÉSULTATS \\ OBJECTIF : \\ AMÉLIORER LA SÉCURITÉ COMMUNAUTAIRE DES \\ POPULATIONS VIVANT DANS DES ZONES AVEC GAC \\ OBJECTIF 1 (ENGAGEMENT) : \\ ACCÈS SÉCURISÉ DES INTERVENANTS AUX ZONES \\ AVEC DES GAC \\ OBJECTIF 2 (GESTION COERCITIVE) : \\ RÉDUCTION DE LA PORTÉE ET DE LA LÉGITIMITÉ \\ DES GAC \\ OBJECTIF 3 (GESTION COOPÉRATIVE) : \\ MEILLEUR TRAITEMENT DE LA POPULATION DANS \\ LES COMMUNAUTÉS LOCALES AVEC DES GAC \\ OBJECTIVE 4 (TRANSFORMATION) : \\ OBSOLESCENCE DES GAC POUR LEURS MEMBRES, \\ COMMANDITAIRES ET COMMUNAUTÉS
}

\section{INDICATEURS}

INDICATEUR 1: POURCENTAGE DE LA POPULATION PERCEVANT DE L'/LA (IN)SÉCURITÉ DANS LEUR COMMUNAUTÉ

INDICATEUR 2 : NOMBRE D'INCIDENTS VIOLENTS SIGNALÉS

INDICATEUR 1.1 : POURCENTAGE DU PERSONNEL DES ACTEURS DE L'HUMANITAIRE ET DU DÉVELOPPEMENT PERCEVANT UN ACCÈS SÉCURISÉ AUX ZONES AVEC DES GAC

\begin{abstract}
INDICATEUR 1.2: NOMBRE ET FRÉQUENCE DES ATTAQUES VISANT LES MEMBRES DU
\end{abstract} PERSONNEL DES ACTEURS DE L'HUMANITAIRE ET DU DÉVELOPPEMENT

INDICATEUR 2.1 : NOMBRE DE PERSONNES VIVANT DANS DES COMMUNAUTÉS AVEC DES GAC INDICATEUR 2.2 : POURCENTAGE DE LA POPULATION PERCEVANT LES GAC COMME LÉGITIMES

INDICATEUR 3.1 : POURCENTAGE DE LA POPULATION PERCEVANT LES GAC COMME DES FORCES POSITIVES ET CONSTRUCTIVES AU SEIN DES COMMUNAUTÉS

INDICATEUR 3.2 : NOMBRE D'INCIDENTS SIGNALÉS DE COMPORTEMENTS ABUSIFS DES GAC À L'ENDROIT DES COMMUNAUTÉS

INDICATEUR 4.1 : NOMBRE DE MEMBRES DES GAC

INDICATEUR 4.2: POURCENTAGE DE LA POPULATION PERCEVANT LA FOURNITURE DE SÉCURITÉ PAR L'ÉTAT COMME SUFFISANTE AU SEIN DES COMMUNAUTÉS

Source : auteur.

\section{ENSEIGNEMENTS TIRÉS DES APPROCHES EN MATIËRE D’EGT}

Les acteurs intervenants ne devraient pas exporter des modèles standards, tels qu'un DDR, d'un environnement à un autre. Les interventions devraient tenir compte des variations de contexte et mettre l'accent sur les fonctions prédominantes des GAC ciblés, suite à une analyse approfondie du contexte et d'une cartographie des parties prenantes. En ayant cependant à l'esprit les limitations des ébauches, les conclusions de ce rapport comportent un certain nombre d'implications pour l'engagement, la gestion et la transformation des GAC, précisément en raison de la forte probabilité que les expériences passées auront une incidence.

\section{Enseignements tirés de l'engagement à court terme}

Bien que les GAC contrôlent rarement un territoire d'une manière exhaustive et qu'ils opèrent rarement en l'absence totale de services gouvernementaux, les populations civiles vivant dans les zones où des GAC opèrent souffrent souvent d'un accès limité aux services de base, tels que la sécurité, les soins de santé et l'éducation. Dans ces circonstances, les acteurs nationaux ou internationaux dont le mandat est de fournir des services de base doivent engager des membres des GAC pour obtenir un accès sécurisé à leur zone d'activités pour les 
membres de leur personnel. Certains acteurs intervenants choisissent de fournir des récompenses financières ou en nature aux GAC pour obtenir un accès sécurisé à leur zone d'activités. En Somalie, par exemple, les agences d'aide ont embauché des milices armées basées dans un clan pour pouvoir assurer une protection ou pour transporter des denrées alimentaires vers des zones non sécurisées. ${ }^{58}$ Des recherches ont cependant montré que cela pouvait avoir des effets négatifs, car cela pourrait renforcer les tendances destructrices des GAC. ${ }^{59}$

Pour s'assurer que l'aide rejoigne les segments les plus vulnérables de la population, les acteurs nationaux et internationaux pourraient essayer de pénétrer dans le fief des GAC par le biais des représentants de la société civile étant donné que ces acteurs appellent largement l'autorité, même vis-à-vis des groupes armés. Il y a toutefois souvent une distinction très nette entre les organisations de la société civile pacifique et les criminels armés, tel que cela est illustré par les comités d'eau potable de district en Somalie agissant au nom des chefs de guerre plutôt que représentant leurs communautés. ${ }^{60}$ Certains acteurs intervenants ont décidé de ne travailler qu'avec des organisations non gouvernementales (ONG) enregistrées auprès des ministères en charge de leur autorisation ; cependant, cette approche peut être problématique car elle pourrait exclure les organisations crédibles de la société civile qui ont été interdites pour des raisons politiques. ${ }^{61}$

Une autre approche est d'inclure les GAC dans les plateformes communautaires en tant que représentants d'un secteur parmi d'autres dont le commerce, l'éducation, la santé, la culture et la religion. Ce faisant, les leaders des GAC peuvent avoir l'impression que leur domination sur leur territoire et que les fonctions qu'ils assument sont respectées ; dans la réalité cependant, ces leaders sont écartés par d'autres acteurs qui dominent le processus de prise de décisions au sein des plateformes. Dans la même veine, les acteurs ayant une relation existante avec les GAC peuvent faciliter l'accès sécurisé afin que d'autres acteurs soient en mesure de mettre en œuvre des projets de développement et d'offrir de l'aide humanitaire. À titre d'exemple, l'ONG internationale Medair a utilisé des intermédiaires bien connectés, comme des professionnels de la santé et des dirigeants traditionnels, pour recueillir des informations sur la sécurité et négocier avec les militaires et les groupes armés afin que ses employés aient accès et puissent fournir de l'aide dans les zones touchées par un conflit dans la province de l'Ituri en République démocratique du Congo. ${ }^{62}$

Outre l'établissement de plateformes par les acteurs de l'humanitaire et du développement, les communautés utilisent ou réinstaurent régulièrement des institutions traditionnelles de médiation de conflit pour faire participer les GAC et améliorer la cohésion de la communauté, souvent avec le soutien des autorités de l'État. ${ }^{63}$ De telles structures traditionnelles de médiation de conflit communautaires ont pour but de promouvoir la réconciliation, de renforcer la confiance réciproque entre les communautés et de prévenir les conflits locaux entre les GAC. Dans l'est de la République démocratique du Congo, par exemple, une initiative gouvernementale a réinstauré de grandes réunions communautaires appelées baraza pour discuter des questions entre représentants de différentes communautés et élire des comités locaux pour la paix. Cependant, si les comités baraza et de paix ont réussi dans un premier temps à résoudre les conflits ethniques et à réduire les cas de

58 Joakim Gundel, Action humanitaire dans le nouvel environnement de sécurité : implications politiques et opérationnelles en Somalie et au Somaliland (Humanitarian Action in the New Security Environment: Policy and Operational Implications in Somalia and Somaliland) (Londres : Overseas Development Institute, 2006).

59 Moritz Schuberth, Faire grandir la base populaire ou soutenir des bandits ? Dilemmes du soutien des donateurs pour la société (non) civile d'Haïti (Growing the Grassroots or Backing Bandits? Dilemmas of Donor Support for Haiti's (Un)Civil Society), Journal of Peacebuilding \& Development $11, \mathrm{n}^{\circ} 1$ (2016) : 93-98.

60 Gundel, Action humanitaire dans le nouvel environnement de sécurité (Humanitarian Action in the New Security Environment), 46.

61 Moritz Schuberth, Engager ou ne pas engager les groupes urbains armés d'Haïti ? Accès sécuritaire aux villes touchées par les catastrophes et les conflits (To Engage or Not to Engage Haiti's Urban Armed Groups? Safe Access in Disaster-Stricken and Conflict-affected Cities), Environment and Urbanization $29, n^{\circ} 2$ (2017) : 425-42.

62 Pottier, L'ethnographie comme barrière (Roadblock Ethnography), 166.

63 Verkore, Willems, Kleingeld, et Rouw, De DDR à promotion de la sécurité (From DDR to Security Promotion), 5. 
violence ethnique, ils se sont effondrés en raison de conflits internes et de la perception communautaire qu'ils étaient sous l'influence de groupes armés. ${ }^{64}$ Par conséquent, il est important que les institutions au niveau de la communauté faisant participer les GAC ne soient pas perçues comme étant trop proches de tels groupes, à défaut de quoi ils perdront leur légitimité auprès de la population.

\section{Enseignements tirés de la gestion à moyen terme}

Comparativement à l'engagement à court terme des GAC, les approches de gestion vis-à-vis des GAC suivent une logique à moyen terme et envisagent un changement de fond des comportements ou de la nature des groupes ciblés. Les approches de gestion coercitive impliquent des forces de sécurité et des mesures musclées pour vaincre les GAC ou incarcérer leurs membres, par exemple des raids de police militarisés contre des gangs en Afrique du Sud. ${ }^{65}$ Les acteurs adoptant des approches de gestion coopérative, en revanche, ont pour but d'altérer les comportement et les rôles des GAC. De tels changements peuvent être obtenus en négociant avec les GAC, comme cela a été le cas avec les grands trafiquants au Mali ; en assurant une médiation entre les GAC, comme cela a été le cas en République centrafricaine entre les rebelles ex-Séléka et les groupes d’autodéfense anti-balaka ; ou en co-optant les GAC, comme cela a été le cas au Nigeria avec la FICC. ${ }^{66}$

\section{APPROCHES COERCITIVES}

Lorsque les GAC sont perçus comme une menace pour l'État, des approches de gestion coercitive par les forces de sécurité de l'État peuvent inclure des raids musclés, ainsi que l'incarcération massive des membres présumés des GAC. Les forces de sécurité ayant recours à des méthodes de pacification ou de contre-insurrection (COIN) - parfois appliquées de manière coercitive pour cibler la fonction politique des GAC - ont pour but de conquérir « les cœurs et les esprits " en " faisant concurrence aux insurgés pour influencer et contrôler au niveau de la base populaire. ${ }^{67}$ La COIN utilisée de cette manière est perçue principalement comme une " stratégie politique " qui doit mettre l'accent sur la construction de la « légitimité politique et l'efficacité - aux yeux de ses populations et de la communauté internationale - d'un gouvernement touché par une insurrection. ${ }^{68}$

Les GAC avec une relation moins antagoniste avec l'autorité de l'État peuvent cependant être également confrontés à de la coercition. En Côte d'Ivoire, par exemple, les chasseurs traditionnels du Nord appelés dozos ont subi une transition approuvée par l'État vers une force de sécurité privée nationale, mais ils ont ensuite été interdits lorsqu'ils ont été impliqués dans des luttes de pouvoir au niveau national. ${ }^{69}$

Les approches coercitives pour les GAC, telles que les opérations de COIN ou les incarcérations de masse, comportent d'importantes limitations. Les communautés locales se sentiront encore plus aliénées si leur expérience des agences étatiques se limitent aux raids militaires qui entraînent la mort de civils en raison de balles perdues. Ce résultat peut avoir l'effet inattendu de pousser les populations locales à soutenir ou

64 Clark, Ethnicité, leadership et médiation de conflit dans l'est de la République démocratique du Congo (Ethnicity, Leadership and Conflict Mediation in Eastern Democratic Republic of Congo), 8.

65 Lamb, Militarisation de la police et la guerre contre la criminalité en Afrique du Sud (Police Militarisation and the 'War on Crime' in South Africa).

66 Oluwaseun Bamidele, Force d'intervention civile conjointe (FICC) - une option de sécurité communautaire : une approche compréhensive et proactive pour réduire le terrorisme (Civilian Joint Task Force'(CJTF)-A Community Security Option: A Comprehensive and Proactive Approach of Reducing Terrorism), Journal for Deradicalization 7 (2016) : 124-44.

67 David Kilcullen, La guérilla accidentelle : mener de petites guerres au milieu d'une grosse (The Accidental Guerrilla: Fighting Small Wars in the Midst of a Big One) (Oxford : Oxford University Press, 2009), xv.

68 Ibid., 265.

69 Lauren Van Metre, D’auto-défenseurs à justiciers : un cadre typologique pour les groupes armés communautaires (From Self-Defense to Vigilantism: ATypology Framework of Community-Based Armed Groups), Washington DC, Réseau RESOLVE, 2019). 
à se joindre aux GAC « parce qu'elles sont aliénées par les actions musclées de la force intervenante. " ${ }^{70}$ En conséquence, les approches coercitives peuvent par inadvertance contribuer à aliéner encore davantage les communautés marginalisées dans un État délégitimisé, permettant ainsi aux GAC de se positionner comme les défenseurs légitimes des communautés contre les forces abusives de l'État. Par exemple, les gangs organisés dans le quartier de Cape Flats du Cap en Afrique du Sud fournissent une protection aux résidents contre les gangs rivaux. Beaucoup perçoivent ces gangs comme plus légitimes que les forces de l'ordre, associées à des raids violents qui entraînent la mort de jeunes locaux. ${ }^{71}$

Le recentrage des efforts des secteurs de la sécurité et de la justice pour lutter contre la grande criminalité organisée et les sections corrompues de l'élite politique qui instrumentalisent les GAC à leur profit s'est avéré plus efficace à long terme que le simple fait de contenir des sections marginalisées de la société. ${ }^{72}$ De plus, une fonction clé de la COIN est la formation de forces de sécurité efficaces et légitimes qui sont capables de fournir " une sécurité centrée sur la population ${ }^{73}{ }^{73}$ En pratique, pour faire cela, il est nécessaire de " créer des populations qui s'autodéfendent par le biais de mesures de sécurité basées sur la communauté, telles que les surveillances du voisinage et les gardes locaux. " ${ }^{74}$ Or, le recours aux GAC en tant que solution provisoire d'application de la loi ou en tant que forces de défense informelles contre les GANE peut de manière non intentionnelle contribuer à la délégitimisation de l'État et à la légitimisation d'acteurs abusifs qui manquent de mécanismes de surveillance, de responsabilité ou de contrôles internes et qui, par conséquent, ont tendance à se retourner contre leurs propres communautés.

\section{LES APPROCHES COOPÉRATIVES}

La collaboration entre les GAC et l'État dans le cadre des interventions de COIN n'est qu'un exemple des multiples types de liens entre les GAC et les acteurs de l'État. Les représentants du gouvernement du Kenya au Zimbabwe embauchent régulièrement les GAC pour attaquer ou intimider leurs opposants ou pour promouvoir leur programme politique personnel en répandant l'insécurité. Les gouvernements pourraient également tolérer, voire encourager, les GAC en tant que mécanismes rentables de contrôle de la criminalité dans les zones de statut de l'État restreint, comme cela s'est produit avec les Sungusungu en Tanzanie. ${ }^{75}$ Dans certains États touchés par un conflit, l'État a coopté les GAC ou les GAC ont agi comme défenseurs autoproclamés de l'État contre d'autres types de GANE, y compris contre des groupes extrémistes violents tels que Boko Haram. Pour renforcer leur légitimité, les GAC peuvent cacher leurs liens avec l'État ou proclamer un degré de proximité avec l’État qui va au-delà de ce que l'État reconnaîtrait officiellement.

Lorsqu'ils sont promus au rang d'agents de la gouvernance hybride de la sécurité, les GAC sont perçus comme la meilleure option disponible pour assurer des services de sécurité et de justice efficaces et légitimes à court terme; à ce titre, ils ont la latitude pour combler l'absence de sécurité laissée par un État absent. Dans de nombreux cas dans la région de l'Afrique subsaharienne, les efforts pour coopter les GAC donnent en définitive la légitimité aux acteurs armés prédateurs et antilibéraux, tandis qu'ils ont délégitimisé l'État et les autres acteurs intervenant qui risquent d'être perçus comme soutenant des criminels. Les exemples vont du Kenya, où les justiciers anti-criminalité ont été coopté par les

70 Kilcullen, La guérilla accidentelle (The Accidental Guerilla), 38.

71 Petrus et Kinnes, Nouveaux bandits sociaux? (New Social Bandits?), 189.

72 Moritz Schuberth, Au-delà des trêves des gangs et les politiques de « main de fer » : vers une gouvernance substitutive de la sécurité en Amérique latine (Beyond Gang Truces and Mano Dura Policies: Towards Substitutive Security Governance in Latin America), Stability: International Journal of Security \& Development 5, $\mathrm{n}^{\circ} 1$ (2016).

73 Kilcullen, La guérilla accidentelle (The Accidental Guerilla), 265.

74 Ibid.

75 Suzette Heald, Contrôler la criminalité et la corruption d'en bas : les Sungusungu au Kenya (Controlling Crime and Corruption from Below: Sungusungu in Kenya), International Relations 21, n² 2 (2007) : 183-99. 
principaux partis politiques et transformés en voyous politiques et en gangs criminels, ${ }^{76}$ à la République démocratique du Congo, où la promesse de positions lucratives pour les leaders de Mai Mai qui ont rejoint les forces de sécurité de l'État a donné naissance à des structures incitatives qui ont favorisé la prolifération et l'expansion des GAC et qui ont entraîné une augmentation de la violence. ${ }^{77}$

II y a cependant des exemples de GAC qui fournissent des services de sécurité sans se transformer en sources d'insécurité. Le rapport de RESOLVE sur les origines de la mobilisation des communautés armées montre, par le biais du cas des Arrow Boys de Teso dans l'est de l'Ouganda, qu'une surveillance dès le début par l'armée nationale peut limiter les abus des GAC. ${ }^{78}$ Au Kenya, il a été montré que les groupes d'autodéfense basés sur la foi - par exemple, les Al Safa nubiens de Kiberia ou un groupe d'autodéfense de lutte contre le trafic de stupéfiants établi par le conseil des imams à Mombasa- ont de solides bases religieuses et culturelles et sont plus résistants à l'instrumentalisation par des barons de la drogue que les GAC laïques. ${ }^{79}$ Un autre exemple est la tentative par un ancien agent de police de superviser les justiciers du bidonville de Kigera à Nairobi en les embauchant au sein d'une entreprise privée de sécurité. Cette pratique a fourni aux membres de ces groupes des salaires réguliers et les a intégrés dans un cadre de règles claires et applicables en formalisant leur rôle de fournisseurs de sécurité, en supprimant ainsi l'incitation à rejoindre un GAC, en leur fournissant un emploi et en appliquant une réglementation. ${ }^{80}$

Le dénominateur commun de ces cas est que la présence de procédures de supervision, un cadre juridique contraignant et des mécanismes de reddition de comptes peut aider à prévenir la transformation des GAC en sources prédatrices d'insécurité. Ces expériences sont conformes aux conclusions présentées dans le document de cartographie de la typologie USIP sur les GAC qui montre que la discipline de groupe et l'acceptation de normes, de rôles et de processus formalisés sont essentielles pour empêcher les GAC de s'engager dans une violence non réglementée, réactive ou opportuniste. ${ }^{81}$ Les interventions externes peuvent renforcer la formalisation des structures de commandement et de contrôle qui institutionnalisent et légitimisent les normes et les règles, par exemple, par la socialisation découlant des croyances partagées, et par des normes organisationnelles et codes de conduite ou des mécanismes de responsabilité et de supervision clairement établis. Le tableau 5 de cette section sur les tendances actuelles et les nouvelles orientations identifie les facteurs de réussite pour accentuer les aspects productifs des GAC et les orienter dans la bonne direction.

\section{Enseignements tirés de la transformation à long terme}

\section{LA RÉFORME DU SECTEUR DE LA SÉCURITÉ}

La réforme du secteur de la sécurité (RSS) suit une logique transformatrice et, vise principalement à remplacer les GAC par des forces de sécurité de l'État fonctionnelles ou à canaliser leurs membres vers ces forces de l'État. Les tentatives ratées pour intégrer les GAC dans les forces de sécurité de l'État, comme cela s'est produit avec les Mai Mai de l’est de la République démocratique du Congo, ont montré

76 David M. Anderson, Justiciers, violence et politiques de maintien de l'ordre au Kenya (Vigilantes, Violence and the Politics of Public Order in Kenya), African Affairs 101, n 405 (2002) : 531-55.

77 Maria Eriksson Baaz et Judith Verweijen, La volatilité d'une bouillabaisse mi-cuite : I'intégration des rebelles militaires et les dynamiques du conflit dans l'est de la RDC (The Volatility of a Half-Cooked Bouillabaisse: Rebel-Military Integration and Conflict Dynamics in the Eastern DRC), African Affairs 112, $n^{\circ} 449$ (2013) : 563-82.

78 Daniel Agbiboa, Dynamiques et mœurs des groupes armés communautaires en Afrique subsaharienne (Dynamics and Drivers of Community-Based Armed Groups in Sub-Saharan Africa) (Washington, DC : Réseau RESOLVE, 2019).

79 Moritz Schuberth, L'impact du trafic de drogue sur les acteurs informels de la sécurité dans l'ensemble du Kenya (The Impact of Drug Trafficking on Informal Security Actors in Kenya), Africa Spectrum 49, $n^{\circ} 3$ (2014) : 55-81.

80 Ibid.

81 Van Metre, D’auto-défenseurs à justiciers (From Self-Defense to Vigilantism). 
que la RSS est autant un processus politique qu'une approche technique ; à ce titre, elle doit tenir compte de la politique locale. ${ }^{82}$ Étant donné les objectifs à long terme et vastes de la RSS, il n'est pas suffisant de former pour obtenir des forces de police plus efficaces et d'endiguer la corruption chez les membres du système judiciaire, aussi difficiles que soient ces initiatives. ${ }^{83}$ Les initiatives de RSS doivent être menées à bien en faisant la promotion de l'État de droit ; les forces de sécurité de l'État doivent être assujetties à une supervision démocratique et civile et tenues responsables de se conformer à des lois alignées aux normes internationales en ce qui concerne les droits humains. ${ }^{84}$ En outre, pour améliorer leur légitimité et leur acceptation parmi les différentes volets de la population du pays d'accueil, les initiatives de RSS doivent accorder une attention particulière à l'appropriation locale, tant par rapport aux élites politiques que par rapport aux communautés dans lesquelles la sécurité est assurée. ${ }^{85}$

Plus concrètement, des tribunaux spéciaux ou des tribunaux nationaux et internationaux peuvent traiter les crimes de guerre ou les groupes de crime organisé transnationaux peuvent gérer les cas politiquement sensibles pour gérer les relations patron-client entre les GAC et leurs commanditaires. Par exemple, le tribunal spécial pour la Sierra Leone a condamné deux anciens dirigeants des Forces de défense civile, un GAC composé de chasseurs traditionnels qui défendaient leurs communautés pendant la guerre civile, mais qui étaient aussi accusés de crimes de guerre et de crimes contre l'humanité. ${ }^{86}$ De plus, la RSS peut renforcer les efforts pour accroître la fourniture de sécurité dans des zones anciennement abandonnées par les agences d'application de la loi et du maintien de l'ordre, où les GAC assumaient les rôles d'agences de contrôle du crime et d'autodéfense. Les acteurs intervenant peuvent le faire en renforçant la capacité et la légitimité de la police et de la justice grâce au renforcement des capacités et à la formation à des techniques adaptées au contexte, comme la police communautaire ou la police de proximité. ${ }^{87}$

\section{DÉSARMEMENT, DÉMOBILISATION ET RÉINTÉGRATION}

Le désarmement, la démobilisation et la réintégration (DDR) est un autre ensemble d'outils et d'approches qui a été discuté dans une vaste littérature universitaire et axée sur les politiques portant sur les leçons techniques et opérationnelles apprises. ${ }^{88}$ De telles directives fournissent des orientations utiles pour le désarmement, qui est sans doute l'aspect le plus simple et le plus technique du DDR. ${ }^{89}$ Pourtant, comme l'ont montré les premières tentatives de DDR en Angola et au Mozambique dans les années 1990, le DDR est une entreprise intrinsèquement politique qui doit être éclairée par une bonne compréhension de l'économie politique des sociétés post-conflit. ${ }^{90}$ La nature politique du DDR est notamment visible

82 Baaz et Verweijen, La volatilité d'une bouillabaisse mi-cuite (The Volatility of a Half-Cooked Bouillabaisse).

83 Adedeji Ebo, Le rôle de la réforme du secteur de la sécurité dans le développement durable : tendances et défis des politiques des donateurs (The Role of Security Sector Reform in Sustainable Development: Donor Policy Trends and Challenges), Conflict, Security \& Development 7, $\mathrm{n}^{\circ} 1$ (2007) : 27-60.

84 Louis-Alexandre Berg, Pistolets, lois et politique : les fondements politiques de l'État de droit et de la réforme du secteur de la sécurité (Guns, Laws and Politics: The Political Foundations of Rule of Law and Security Sector Reform), Hague Journal on the Rule of Law 4, n 1 (2012): 4-30.

85 Eleanor Gordon, Réforme du secteur de la sécurité, appropriation locale et engagement de la communauté (Security Sector Reform, Local Ownership and Community Engagement), Stability: International Journal of Security \& Development 3, $n^{\circ} 1$ (2014) : 14. 25.

86 Danny Hoffman, La signification de la milice : comprendre les forces de défense civile de la Sierra Leone (The Meaning of a Militia: Understanding the Civil Defence Forces of Sierra Leone), African Affairs 106, n 425 (2007) : 639-62.

87 Schuberth, Au-delà des trêves de gangs (Beyond Gang Truces).

88 Nations Unies, Guide opérationnel pour des normes intégrées sur le désarmement, la démobilisation et la réintégration (Operational Guide to the Integrated Disarmament, Demobilization and Reintegration Standards), New York : Nations Unies, 2010 ; Tatjana Stankovic et Stina Torjesen, Nouvelles réflexions sur le désarmement, la démobilisation et la réintégration : sondage à l'intention des praticiens (Fresh Insights on Disarmament, Demobilization and Reintegration: A Survey for Practitioners) (Oslo : Norwegian Institute of International Affairs, 2010).

89 Voir par exemple Savannah De Tessières, Gestion efficace des armes et des munitions dans un contexte changeant désarmement, démobilisation et réintégration : un manuel pour les praticiens du DDR des Nations Unies (Effective Weapons and Ammunition Management in a Changing Disarmament, Demobilization and Reintegration Context : A Handbook for United Nations DDR Practitioners) (New York : Département des opérations de maintien de la paix des Nations Unies et Bureau des affaires de désarmement des Nations Unies, 2018).

90 Mats Berdal et David Ucko, eds., Réintégrer les groupes armés après un conflit : politique, violence et transition (Reintegrating Armed Groups After Conflict: Politics, Violence and Transition) (New York : Routledge, 2009). 
lors de la démobilisation, qui vise à remplacer la relation patron-client entre les « entrepreneurs de violence " au sein de l'élite politique et les anciens combattants en démantelant les anciennes structures de commandement et en brisant les liens de la hiérarchie. ${ }^{91}$

Comme l'ont montré des recherches en République du Congo et en Sierra Leone, les anciens commandants de niveau intermédiaire jouent souvent un rôle important d'intermédiaires entre l'élite et les membres démobilisés des groupes armés; leur influence sur leurs anciens subordonnés reste souvent suffisamment forte pour pouvoir les remobiliser efficacement. ${ }^{92}$ En outre, le calendrier du DDR est crucial ; un désarmement et une démobilisation prématurés ne seront pas durables si la menace extérieure qui a conduit à l'émergence des GAC reste en place. Cela est particulièrement vrai pour les GAC protégeant leurs communautés contre les GANE, comme la FICC combattant Boko Haram au Nigeria.

Bien que la réintégration sociale, économique et politique réussie des anciens combattants soit à la fois l'aspect le plus crucial et le plus complexe du DDR, les programmes en pratique se concentrent trop souvent sur la réinsertion à court terme plutôt que sur la réintégration à long terme. ${ }^{93}$ Les programmes de réintégration communautaires visent à améliorer leur durabilité en rassemblant les anciens membres des GAC et leurs communautés pour identifier et travailler ensemble sur des projets qui profitent à l'ensemble des communautés, comme la construction ou la réhabilitation d'infrastructures critiques. ${ }^{94}$ Dans la province de Maniema en République démocratique du Congo, par exemple, des organisations locales de la société civile et Oxfam Novib ont mis en place un projet "d'armes pour le développement ", dans lequel le désarmement volontaire a été récompensé par une aide agricole et la réhabilitation d'écoles et d'un centre de santé. ${ }^{95}$ Cependant, des études ont montré que pour réussir les programmes DDR centrés sur la communauté doivent être véritablement dirigés par la communauté, tout en étant une priorité des bailleurs de fonds nationaux et internationaux. ${ }^{96}$

\section{RÉDUCTION DE LA VIOLENCE COMMUNAUTAIRE}

Bien que les efforts en matière de DDR menés par la communauté soient bien adaptés à la réintégration des anciens membres du GAC, ils ont tendance à négliger ceux d'une communauté qui n'en sont pas devenus membres, mais qui risquent de le faire. Pour surmonter cette limite, les acteurs nationaux et internationaux ont mis en place des programmes opérationnels de réduction de la violence communautaire (RVC) qui peuvent être décrits comme des programmes communautaires de DDR intégrant des aspects de réduction et de prévention de la violence armée ou RPVA (armed violence reduction and prevention ou AVRP). ${ }^{97}$ La RPVA se concentre sur l'identification des facteurs de risque « qui contribuent à augmenter la probabilité qu'une personne commette un acte violent " et des facteurs de résilience " qui aident les personnes dans des circonstances défavorables à surmonter l'adversité et à éviter la violence $"{ }^{98}$ Avec

91 Anders Themnér, Acte de foi : quand et comment les anciens combattants ont recours à la violence (A Leap of Faith: When and ow Ex-Combatants Resort to Violence), Security Studies 22, nº 2 (2013) : 295-329.

92 Ibid.

93 Alpaslan Özerdem, Une reconceptualisation de la réintégration des anciens combattants: une approche de la «réintégration sociale » (Re-Conceptualisation of Ex-Combatant Reintegration: 'Social Reintegration' Approach), Conflict, Security \& Development 12, $\mathrm{n}^{\circ} 1$ (2012) : 51-73.

94 Prosper Nzekani Zena, Les enseignements et les limites du DDR en Afrique (The Lessons and Limits of DDR in Africa) (Washington, DC : Africa Center for Strategic Studies, 2013), 7.

95 Verkoren, Willems, Kleingeld et Rouw, De DDR à la promotion de la sécurité (From DDR to Security Promotion), 19.

96 Özerdem, Une reconceptualisation de la réintégration des anciens combattants (A Re-conceptualisation of Ex-combatant Reintegration), 59 ; Julia Buxton, Réintégration et développement à long terme : liens et défis (Reintegration and Long-term Development: Linkages and Challenges) (Bradford : Centre for International Cooperation and Security, 2008), 23.

97 Verkoren, Willems, Kleingeld et Rouw, De DDR à la promotion de la sécurité (From DDR to Security Promotion).

98 Jennifer M. Hazen, Risque et résilience : comprendre le potentiel de violence (Risk and Resilience: Understanding the Potential for Violence), dans Small Arms Survey 2008 (Genève : Small Arms Survey), 2008, 245. 
I'identification de ces deux ensembles de facteurs, les interventions ciblées peuvent se concentrer sur l'atténuation des risques pour prévenir la survenue de violence armée.

Les efforts de RVC font partie des approches innovantes de deuxième génération pour faire face à la violence armée qui se concentrent sur la résolution de l'ensemble complexe des causes profondes de la violence et de l'insécurité au niveau local. ${ }^{99}$ Pour ce faire, la RVC vise à stabiliser les communautés à court terme en fournissant des moyens de revenu alternatifs aux jeunes à risque tout en améliorant la cohésion communautaire à long terme. La RVC peut donc être considérée comme une approche globale du développement communautaire qui offre des alternatives aux jeunes risquant de rejoindre les GAC, en remplaçant les fonctions que ces groupes remplissent pour leurs membres, comme la protection et l'accès aux ressources et un statut plus élevé. La Mission multidimensionnelle intégrée des Nations Unies pour la stabilisation en République centrafricaine (MINUSCA), par exemple, a fourni aux jeunes chômeurs un emploi temporaire et une formation professionnelle pour les éloigner des opportunités économiques offertes par les GAC. Un facteur clé de succès a été d'inclure les communautés elles-mêmes dans la planification, la mise en œuvre et le suivi des projets de RVC. ${ }^{100}$

La grande attention accordée par la RVC à l'emploi temporaire par le biais de programmes de trésorerie pour le travail a été critiquée comme étant non viable et sujette à la corruption et à l'exploitation. ${ }^{101}$ Une autre critique est que le champ thématique des initiatives de RVC est trop large, qu'elles sont déconnectées les unes des autres et, dans de nombreux cas, qu'elles ne sont pas clairement liées à l'objectif ultime de réduction de la violence. ${ }^{102}$ En même temps, le large champ thématique des interventions dans des communautés géographiquement bien délimitées est précisément ce qui distingue la RVC des expériences traditionnelles et souvent infructueuses de DDR. Les DDR traditionnels se concentrent sur des groupes particuliers, tandis que la RVC a une orientation géographique clairement définie. En conséquence, la RVC pourrait être plus applicable dans des contextes où des GAC avec des hiérarchies faibles et des structures organisationnelles souples sont présents, tandis que le DDR pourrait être mieux adapté aux groupes armés avec un commandement hiérarchique strict. ${ }^{103}$

\section{TENDANCES ACTUELLES ET NOUVELLES ORIENTATIONS}

\section{Les GAC et la légitimité de l'État}

Comme discuté, le prisme de la fragilité de l'État n'offre pas une explication universelle à la prolifération des GAC. Dans certains cas, un manque de légitimité étatique plutôt qu'un manque de capacité

99 Robert Muggah et Francis Sang, L'ennemi à l'intérieur : repenser la disponibilité des armes en Afrique subsaharienne (The Enemy Within: Rethinking Arms Availability in Sub-Saharan Africa), Conflict, Security \& Development 13, $n^{\circ} 4$ (2013) : 437 ; Robert Muggah et Keith Krause, Combler l'écart entre les opérations de paix et l'insécurité post-conflit : vers un programme de réduction de la violence (Closing the Gap Between Peace Operations and PostConflict Insecurity: Towards a Violence Reduction Agenda), International Peacekeeping 16, $n^{\circ} 1$ (2009) : 136-50.

100 Robert Muggah, Les Nations Unies ne peuvent pas apporter la paix en République centrafricaine (The U.N. Can't Bring Peace to the Central African Republic), Foreign Policy, 16 août 2018, https://foreignpolicy.com/2018/08/16/the-u-n-cant-bring-peace-to-the-central-african-republic.

101 Timothy Donais, Revenir au local : Haïti, gouvernance locale et dynamiques de la construction verticalement intégrée de la consolidation de la paix (Bringing the Local Back In: Haiti, Local Governance and the Dynamics of Vertically Integrated Peacebuilding), Journal of Peacebuilding \& Development $10, \mathrm{n}^{\circ} 1(2015): 46$.

102 Ibid., 45.

103 Brian McQuinn, DDR et l'organisation interne des groupes armés non étatiques (DDR and the Internal Organization of Non-State Armed Groups), Stability: International Journal of Security \& Development $5, \mathrm{n}^{\circ} 1$ (2016). 
institutionnelle contribue à la formation des GAC. En même temps, les fonctions sécuritaires, politiques et économiques que les GAC remplissent pour diverses parties prenantes peuvent avoir des effets à la fois de légitimation et de délégitimation. Des recherches sur les GAC au Kenya montrent que si la fonction sécuritaire est légitimante, l'élément économique tend à être plus délégitimisant ; l'impact du facteur politique sur la légitimité des GAC est détourné en fonction de critères ethniques. ${ }^{104}$

En assurant la sécurité dans les zones à statut restreint que les agences d’application de la loi négligent, les GAC kényans comblent un vide institutionnel et gagnent en légitimité. En revanche, ils sont connus pour extorquer de l'argent aux habitants en échange de leur protection, ce qui diminue leur légitimité. ${ }^{105}$ Dans le même temps, il a été montré que les GAC au Kenya travaillent sous les ordres de groupes criminels organisés pour des raisons intéressées, ce qui compromet encore leur propre légitimité ${ }^{106}$ Enfin, les entrepreneurs ethniques ont manipulé et instrumentalisé les GAC comme outils politiques pour attaquer les membres de groupes ethniques rivaux au moment des élections. Cela conduit à leur délégitimation complète parmi les personnes visées par les attaques; cependant, cela contribue à leur légitimité parmi les pairs ethniques qu'ils protègent contre des attaques similaires de milices rivales. ${ }^{107}$

\section{De GAC à fournisseurs de sécurité communautaire?}

Le rapport de recherche USIP/RESOLVE sur les dynamiques et les moteurs des GAC a montré que la gouvernance hybride de la sécurité est une réalité dans de nombreuses régions d'Afrique subsaharienne et que la fourniture plurielle de sécurité peut se manifester de manière plus constructive ou plus destructrice. ${ }^{108}$ Le document de cartographie de la typologie USIP/RESOLVE sur les GAC a souligné comment la trajectoire des GAC est influencée par des facteurs internes, tels que leur structure organisationnelle, ainsi que par des facteurs externes, tels que les normes et l'ordre social. ${ }^{109}$ Ce document de cartographie a identifié un certain nombre d'études de cas dans lesquelles les justiciers ne se sont pas transformés en milices politiques ou en criminels prédateurs en raison de facteurs internes, tels que des règles applicables, et de facteurs externes, tels qu'une surveillance étatique forte ou un cadre juridique favorable. Les acteurs intervenants peuvent ainsi influencer la trajectoire des GAC et orienter leur comportement dans la direction souhaitée. Ce processus est cependant loin d'être simple car de multiples facteurs peuvent influencer le comportement des GAC et les interventions en matière d'EGT peuvent avoir des conséquences involontaires, surtout si elles ne sont pas adaptées de manière adéquate à des contextes spécifiques.

Le tableau 5 répertorie les facteurs de succès potentiels que les acteurs intervenants peuvent prendre en compte lors de la conception de stratégies visant à modifier les caractéristiques internes et externes des GAC pour renforcer leur potentiel constructif et limiter leur potentiel destructeur.

104 Moritz Schuberth, Gouvernance hybride de la sécurité, violence post-conflit et légitimité des groupes armés communautaires dans les zones urbaines du Kenya (Hybrid Security Governance, Post-election Violence and the Legitimacy of Community-based Armed Groups in Urban Kenya), Journal of Eastern African Studies 12, $\mathrm{n}^{\circ} 2$ (2018) : 386-404.

105 Erik Henningsen et Peris Jones, "Quel genre d'enfer cela est-il ? " Comprendre la puissance de mobilisation des Mungiki (What Kind of Hell is This! Understanding the Mungiki Movement's Power of Mobilisation), Journal of Eastern African Studies 7, $n^{\circ} 3$ (2013) : 372.

106 Schuberth, L'impact du trafic de drogue sur les acteurs informels de la sécurité dans l'ensemble du Kenya (The Impact of Drug Trafficking on Informal Security Actors in Kenya).

107 Schuberth, Gouvernance hybride de la sécurité (Hybrid Security Governance).

108 Agbiboa, Dynamiques et mœurs des groupes armés communautaires en Afrique subsaharienne (Dynamics and Drivers of Community-Based Armed Groups in Sub-Saharan Africa).

109 Van Metre, D’auto-défenseurs à justiciers (From Self-Defense to Vigilantism). 


\section{Tableau 5 : Facteurs de la transformation des GAC en prestataires de sécurité communautaire}

\begin{tabular}{|c|c|c|c|}
\hline & $\begin{array}{c}\text { ENVIRONNEMENT } \\
\text { JURIDIQUE ET } \\
\text { RÉGLEMENTAIRE }\end{array}$ & $\begin{array}{c}\text { CAPACITÉ } \\
\text { ORGANISATIONNELLE }\end{array}$ & $\begin{array}{l}\text { RESPONSABILITÉ ET } \\
\text { SUPERVISION }\end{array}$ \\
\hline $\begin{array}{r}\text { FACTEUR } \\
\text { DE SUCCÈS } \\
1\end{array}$ & $\begin{array}{l}\text { IL EXISTE DES } \\
\text { LOIS CLAIRES SUR } \\
\text { L'ENREGISTREMENT } \\
\text { DES GAC ET LES GAC } \\
\text { S'ENREGISTRENT } \\
\text { SANS OBSTACLES. }\end{array}$ & $\begin{array}{l}\text { LES GAC ONT ET SUIVENT } \\
\text { DES MISSIONS ET } \\
\text { OBJECTIFS CLAIREMENT } \\
\text { DÉFINIS. }\end{array}$ & $\begin{array}{l}\text { LES GOUVERNEMENTS } \\
\text { LOCAUX ET/OU } \\
\text { CENTRAUX FOURNISSENT } \\
\text { DES FONDS AUX GAC } \\
\text { DE MANIÈRE OUVERTE } \\
\text { ET TRANSPARENTE ET } \\
\text { LES GAC PRENNENT LES } \\
\text { MESURES APPROPRIÉES } \\
\text { POUR ÉVITER LES } \\
\text { CONFLITS D'INTÉRÊTS. }\end{array}$ \\
\hline
\end{tabular}

LES LOIS ET

RÉGLEMENTATIONS

SUR LES GAC

DÉFINISSENT

FACTEUR LES RÔLES ET

DE SUCCÈS RESPONSABILITÉS CLAIRS ET LIMITÉS,

2 Limitant la portéE DE LEURS ACtivités AUTORISÉES.

\author{
LES GAC ONT DES \\ STRUCTURES DE GESTION \\ CLAIREMENT DÉFINIES \\ ET DES POLITIQUES \\ OU PROCÉDURES \\ ÉCRITES POUR GUIDER \\ LES OPÉRATIONS \\ ORGANISATIONNELLES. \\ LES GAC ONT ADOPTÉ \\ ET SUIVENT UN \\ CODE D'ÉTHIQUE, \\ FONCTIONNENT DE \\ MANIÈRE TRANSPARENTE \\ ET FONT L'OBJET \\ D'AUDITS FINANCIERS \\ RÉGULIERS.
}

VIABILITÉ
FINANCIÈRE ET
DURABILITÉ
LES GAC ONT ACCÈS
À DES SOURCES
DE FINANCEMENT
PÉRENNES POUR
POURSUIVRE LEURS
ACTIVITÉS À COURT ET
LONG TERME.

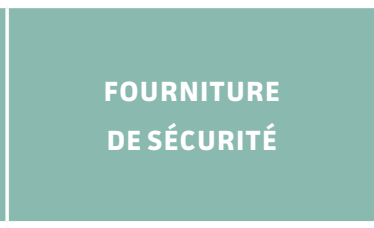

LES SERVICES DE SÉCURITÉ FOURNIS PAR LES GAC REFLÈTENT LES BESOINS ET LES PRIORITÉS DE LEURS COMMUNAUTÉS.
LES GAC LÈVENT

LEUR FINANCEMENT

AUPRÈS DE SOURCES

LOCALES, Y COMPRIS

LES GOUVERNEMENTS,

LES ENTREPRISES ET LES

MEMBRES INDIVIDUELS.
LES GAC ONT DES PROCÉDURES CLAIRES ET TRANSPARENTES EN PLACE POUR DÉTERMINER LES BESOINS ET LES PRIORITÉS DE LEURS COMMUNAUTÉS.
LES LOIS ET RÈGLEMENTS SUR LES GAC SONT MIS EN

FACTEUR DE SUCCÈS

3 GEUVRE DE MANIÈRE COHÉRENTE ET CONFORMÉMENT À LEURS DISPOSITIONS.
LES GAC PEUVENT MAINTENIR DU PERSONNEL PERMANENT et RÉMUNÉRÉ.
LES LOIS ET RÈGLEMENTS FOURNISSENT DES DIRECTIVES CLAIRES SUR LA SUPERVISION GOUVERNEMENTALE DES GAC.

IL EXISTE DES MÉCANISMES DE SURVEILLANCE EXTERNES CLAIRS ET TRANSPARENTS, Y COMPRIS LE GOUVERNEMENT ET LES COMMISSIONS NATIONALES DES DROITS DE L'HOMME.
LES GAC NE DÉPENDENT PAS DE FINANCEMENTS ÉTRANGERS QUI POURRAIENT FLUCTUER EN FONCTION DES NIVEAUX DE FINANCEMENT ET DES PRIORITÉS DES DONATEURS ÉTRANGERS.

LES GAC DISPOSENT DE SYSTÈMES DE GESTION FINANCIÈRE SOLIDES OU ONT ACCÈs À DES SERVICES PROFESSIONNELS DE GESTION FINANCIÈRE.
LES GAC FOURNISSENT LEURS SERVICES DE SÉCURITÉ AUX PERSONNES AU-DELÀ DE LEURS PROPRES MEMBRES ET SANS DISCRIMINATION EN CE QUI CONCERNE LA RACE, LE SEXE, L'ORIGINE ETHNIQUE, L'ORIENTATION SEXUELLE, ETC.
LES GAC RECOUVRENT UNE PARTIE DES COÔTS DE LA PRESTATION DE SERVICES PAR LE BIAIS DE FRAIS VOLONTAIRES, MAIS S'ABSTIENNENT DE FAIRE APPLIQUER LA PERCEPTION DES FRAIS OBLIGATOIRES. 
Pour que ces stratégies aboutissent, elles doivent renforcer et formaliser la fonction sécuritaire des GAC tout en diminuant leurs fonctions politiques et criminelles, sans affaiblir la légitimité de l'État. Les facteurs de succès visent à transformer les GAC multidimensionnels en fournisseurs de sécurité communautaire plus responsables, capables et respectueux des règles qui fonctionnent davantage comme des organisations de la société civile que comme des justiciers informels. ${ }^{110}$ II est toutefois important de garder à l'esprit qu'une telle transformation est difficile à réaliser dans des situations d'urgence politique complexes parce qu'elle est coûteuse et nécessite des engagements à long terme de la part de multiples acteurs.

\section{Le défi de la coordination des interventions en matière d'EGT}

Les urgences politiques complexes, un contexte typique dans lequel les acteurs intervenant utilisent des approches en matière d'EGT pour les GAC, nécessitent une approche globale en raison de leur « nature multi-causale ». Cependant, une telle approche reste un défi étant donné une répartition formelle du travail entre différents acteurs intervenant - tels que les agences de développement ou humanitaires et les forces armées - qui opèrent sur la base de logiques institutionnelles et de visions profondes du monde différentes. Dans le cas de la mission de I'ONU au Soudan, par exemple, les tentatives pour poursuivre une approche internationale intégrée afin de soutenir les efforts nationaux de DDR ont échoué en raison des tensions entre le PNUD et la DPKO, et du manque de volonté politique du gouvernement soudanais. En outre, différents acteurs intervenant montrent des attitudes divergentes concernant la coordination des approches en matière d'EGT. Par exemple, l'armée a tendance à chercher à subordonner et à instrumentaliser d'autres acteurs, tandis que certains acteurs humanitaires ont développé une aversion générale pour l'intégration, estimant qu'elle avait "systématiquement compromis les valeurs fondamentales de leurs organisations $»$.

Le manque de cohérence et de coordination entre la multitude d'acteurs impliqués dans l'EGT des GAC peut avoir de graves répercussions non seulement sur le résultat global des interventions, mais aussi sur la sécurité des acteurs intervenants et des communautés supposées en être les bénéficiaires. Bien que la cohérence et la coordination entre les différentes approches $E, G$ ou $T$ sont difficiles, le défi de la coordination de différents outils au sein d'une même approche $E, G$ ou T peut potentiellement être surmonté par un cadre d'action intégré et cohérent visant simultanément les trois fonctions des GAC : sécurité, économique et politique. Un exemple est celui des approches transformatives pour remplacer les GAC par un État moderne et responsable, lié par l'État de droit et servant toutes les parties de la société de manière juste et équitable. Comme l'illustre la figure 1 , une approche transformative intégrée implique une RSS pour remplacer la fonction de sécurité des GAC, un RDD pour remplacer leur fonction politique et une RVC pour remplacer leur fonction économique.

Le RDD peut aider à rompre la relation descendante patron-client entre les GAC et leurs commanditaires ; la RCV vise à arrêter le flux ascendant de nouvelles recrues des communautés vers les GAC. La RSS peut jouer un rôle complémentaire en contribuant à mettre fin à l'impunité pour l'élite politico-criminelle et à améliorer l'offre de sécurité de l'État dans les zones à statut restreint. Ces stratégies de transformation peuvent être utilement soutenues par certaines approches coercitives et coopératives, comme le ciblage précis des dirigeants du GAC qui résistent aux appels à désarmer et à démobiliser et à fournir un accès sûr aux acteurs humanitaires et du développement. Cependant, les approches de transformation peuvent être potentiellement trop ambitieuses, notamment en tenant compte des délais de mise en œuvre courts

110 Les facteurs de succès sont adaptés à partir des indicateurs présentés dans l'indice de durabilité des organisations de la société civile de USAID (CSOSI) et dans les fiches de référence des indicateurs de démocratie, des droits de l'Homme et de gouvernance de I'USAID. 
et des limitations budgétaires. Une meilleure coordination interorganisations peut aider à mettre en commun les ressources existantes pour améliorer l'efficacité et à diversifier les sources de financement pour optimiser leur durabilité.

Figure 1 : Cadre intégré pour les approches de transformation des GAC

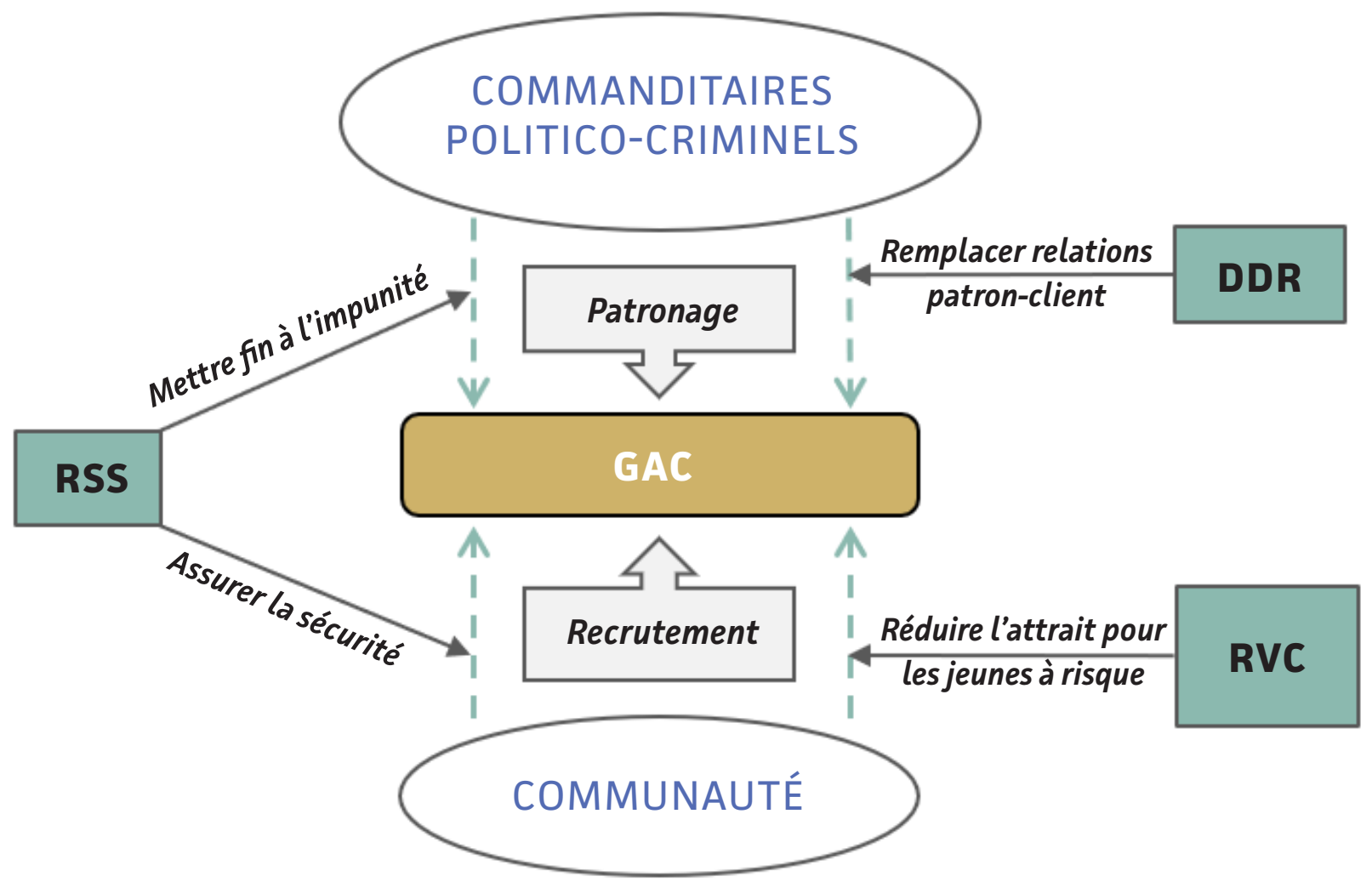

\section{CONCLUSION}

Les GAC se situent dans un espace compliqué entre sécurité et développement, nécessitant des approches communautaires et gouvernementales. Ce document dresse une cartographie des enseignements tirés des approches en matière d'EGT pour les GAC, identifie les facteurs de succès pour transformer les GAC en fournisseurs de sécurité communautaire et propose des indicateurs pour mesurer les progrès des approches EGT par rapport à leurs objectifs. II montre également que, contrairement à une grande partie de la littérature sur la fragilité des États, les GAC sont également présents dans des pays qui jouissent d'une force économique relative et d'un statut d'État fort au sein de leurs régions respectives, mais souffrent d'un niveau élevé d'inégalités sociales, notamment en Afrique du Sud. 
D'importantes limites touchent tous les types d'approches EGT. Les acteurs intervenants tentent fréquemment d'appliquer des outils " universels " qui ont été développés et testés dans des contextes spécifiques à des contextes apparemment similaires, sans tenir compte des différences de contexte et de nature des GAC. En outre, le manque de cohérence et de coordination entre les différents acteurs intervenant conduit régulièrement à la duplication des efforts et à l'utilisation d'approches contradictoires aux GAC individuels. Sur la base des conclusions de cet article, il existe d'importantes leçons supplémentaires à tirer des différentes approches relatives aux GAC dans divers contextes. Voici quelques-unes des questions qui ont émergé et qui devraient être approfondies au moyen d'études de cas nationales :

1. Quels sont les outils d'EGT les plus appropriés pour quel type de GAC (justiciers, milices, gangs) et dans quel contexte (par exemple, urbain contre rural ; présence d'extrémisme violent contre absence d'extrémisme violent) ?

2. Quel échéancier est nécessaire pour les différents outils d'EGT ? Quand la situation est-elle adaptée aux différents types d'approches d'EGT ?

3. Quels sont les intervenants les plus efficaces pour les différents types d'approches d'EGT ? Qui est indispensable pour les différentes approches?

4. De quelle manière des exemples concrets d'approches d'EGT relatives aux GAC ont-ils affecté la légitimité de l'État?

Pour répondre à ces questions, il serait utile de mener des recherches approfondies sur des études de cas spécifiques, y compris parmi les deux principaux complexes de conflits régionaux en Afrique subsaharienne : le bassin du lac Tchad et la région des Grands lacs africains. Par exemple, le Nigeria et la République démocratique du Congo fourniraient une comparaison transnationale d'un État confronté à l'extrémisme violent et d'un autre confronté à des menaces plus pressantes que l'extrémisme violent, même si l'État islamique (EI) a récemment revendiqué ses premiers attentats dans l'est de la République démocratique du Congo. Ces deux études de cas permettraient de comparer dans le pays les réponses aux GAC urbains et ruraux. En outre, l'épidémie d'Ebola dans l'est de la République démocratique du Congo offrirait une occasion unique d'examiner les leçons tirées de l'EGT des GAC dans le contexte d'une épidémie ou d'une crise sanitaire ; elle pourrait conduire à des recommandations de politiques nécessaires en toute urgence sur la manière dont les intervenants nationaux et internationaux peuvent les engager. 


\section{SOURCES}

Abrahams, Raphael Garvin. Citoyens justiciers : l'autodéfense et l'État (Vigilant Citizens: Vigilantism and the State). Malden, Massachusetts : Polity Press, 1998.

Agbiboa, Daniel. Dynamiques et mœurs des groupes armés communautaires en Afrique subsaharienne (Dynamics and Drivers of Community-Based Armed Groups in Sub-Saharan Africa). Washington, DC : Réseau RESOLVE, 2019.

Anderson, David M. Justiciers, violence et politiques de maintien de l'ordre au Kenya (Vigilantes, Violence and the Politics of Public Order in Kenya), African Affairs 101, $\mathrm{n}^{\circ} 405$ (2002) : 531-55.

Baaz, Maria Eriksson et Judith Verweijen. La volatilité d'une bouillabaisse mi-cuite : I'intégration des rebelles militaires et les dynamiques du conflit dans l'est de la RDC, (The Volatility of a Half-Cooked Bouillabaisse: Rebel-Military Integration and Conflict Dynamics in the Eastern DRC), African Affairs 112, $\mathrm{n}^{\circ} 449$ (2013) : 563-82.

Baker, Bruce. Fournisseurs non étatiques de services de sécurité dans les États africains fragiles (Nonstate Providers of Everyday Security in Fragile African States), Fragile States and Insecure People?, édité par Louise Andersen, Bjørn Møller, et Finn Stepputat, 123-47. New York : Palgrave Macmillan, 2007.

Baker, Bruce, et Eric Scheye. Fourniture de justice et de sécurité multi-niveaux dans les États post-conflits et fragiles : une analyse (Multi-Layered Justice and Security Delivery in Post-Conflict and Fragile States: Analysis), Conflit, Security \& Development 7, $\mathrm{n}^{\circ} 4$ (2007) : 503-28.

Bamidele, Oluwaseun. Force d'intervention civile conjointe (FICC) - une option de sécurité communautaire : une approche compréhensive et proactive pour réduire le terrorisme (Civilian Joint Task Force'(CJTF)-A Community Security Option: A Comprehensive and Proactive Approach of Reducing Terrorism), Journal for Deradicalization 7 (2016) : 124-44.

Barnett, Michael N., et Martha Finnemore. La politique, le pouvoir et les pathologies des organisations internationales (The Politics, Power, and Pathologies of International Organization), International Organization 53, $\mathrm{n}^{\circ} 4$ (1999) : 699-732.

Bennett, Will. Manuel de sécurité communautaire (Community Security Handbook). Londres : Saferworld, 2014.

Berdal, Mats, et David Ucko, eds. Réintégrer les groupes armés après les conflits : politique, violence et transition (Reintegrating Armed Groups After Conflict: Politics, Violence and Transition). New York, Routledge, 2009.

Berg, Louis-Alexandre. Pistolets, lois et politique : les fondements politiques de l'État de droit et de la réforme du secteur de la sécurité (Guns, Laws and Politics: The Political Foundations of Rule of Law and Security Sector Reform), Hague Journal on the Rule of Law 4, $\mathrm{n}^{\circ} 1$ (2012) : 4-30.

Boege, Volker, M. Anne Brown et Kevin P. Clements. Des commandes politiques hybrides, pas des États fragiles (Hybrid Political Orders, not Fragile States), Peace Review 21, n 1 (2009) : 13-21.

Boutellis, Arthur. L'ONU peut-elle stabiliser le Mali ? Vers une doctrine de stabilisation de l'ONU ? (Can the UN Stabilize Mali? Towards a UN Stabilization Doctrine?), Stability: International Journal of Security and Development 4, $\mathrm{n}^{\circ} 1$ (2015).

Brown, Oli, Anne Hammill et Robert McLeman. Le changement climatique en tant que « nouvelle » menace à la sécurité : implications pour l'Afrique (Climate Change as the 'New' Security Threat: Implications for Africa), International Affairs 83, ${ }^{\circ} 6$ (2007) : 1141-54.

Buxton, Julia. Réintégration et développement à long terme : liens et défis (Reintegration and Long-Term Development: Linkages and Challenges). Mon Alto, CA : Centre for International Cooperation and Security (2008).

Buzan, Barry, Ole Wæver et Jaap De Wilde. Sécurité : un nouveau cadre d’analyse (Security: A New Framework for Analysis). Boulder, CO : Lynne Rienner Publishers, 1998.

Call, Charles T. La fausse hypothèse de l'« État défaillant » (The Fallacy of the 'Failed State'), Third World Quarterly 29, n 8 (2008) : $1491-1507$.

Clark, Phil. Ethnicité, leadership et médiation de conflit dans l'est de la République démocratique du Congo : le cas des Barza intercommunautaires (Ethnicity, Leadership and Conflict Mediation in Eastern Democratic Republic of Congo: The Case of the Barza Inter-Communautaire), Journal of Eastern African Studies 2, $\mathrm{n}^{\circ} 1$ (2008) : 1-17.

Colak, Alexandra Abello, et Jenny Pearce. Sécurité « d'en bas » dans le contexte de la violence chronique ('Security from Below' in Contexts of Chronic Violence), IDS Bulletin 40, $n^{\circ} 2$ (2009) : 11-19.

De Tessières, Savannah. Une gestion efficace des armes et des munitions dans un contexte changeant de désarmement, de démobilisation et de réintégration : un manuel pour les praticiens du DDR des Nations unies (Effective Weapons and Ammunition Management in a Changing Disarmament, Demobilization and Reintegration Context: A Handbook for United Nations DDR Practitioners). New York : Département des opérations de maintien de la paix des Nations Unies et Bureau des affaires de désarmement des Nations Unies, 2018.

De Vries, Hugo. Les fluctuations de la stabilisation au Congo (The Ebb and Flow of Stabilization in the Congo). Londres/Nairobi : Rift Valley Institute, 2016.

Denney, Lisa. Surmonter la division État/non-État : une approche utilisateur final de la réforme de la sécurité et de la justice (Overcoming the State/NonState Divide: An End User Approach to Security and Justice Reform), International Peacekeeping 21, $\mathrm{n}^{\circ} 2$ (2014) : 251-68.

Dietrich, Kyle. Quand on ne voit pas l'ennemi, les civils deviennent l'ennemi : vivre pendant les six ans d'insurrection au Nigeria (When We Can't See the Enemy, Civilians Become the Enemy: Living Through Nigeria's Six-Year Insurgency). Washington, DC : Center for Civilians in Conflict, 2015. 
Donais, Timothy. Revenir au local : Haïti, gouvernance locale et dynamiques de la construction verticalement intégrée de la consolidation de la paix (Bringing the Local Back In: Haiti, Local Governance and the Dynamics of Vertically Integrated Peacebuilding), Journal of Peacebuilding \& Development 10, $\mathrm{n}^{\circ} 1$ (2015): 40-55.

Duffield, Mark. Développement, sécurité et guerre sans fin : gouverner le monde des populations (Development, Security and Unending War: Governing the World of Peoples). Cambridge : Polity Press, 2007.

Ebo, Adedeji. Le rôle de la réforme du secteur de la sécurité dans le développement durable : tendances et défis des politiques des donateurs (The Role of Security Sector Reform in Sustainable Development: Donor Policy Trends and Challenges), Conflict, Security \& Development 7, $\mathrm{n}^{\circ} 1$ (2007): 27-60.

Gordon, Eleanor. Réforme du secteur de la sécurité, appropriation locale et engagement de la communauté (Security Sector Reform, Local Ownership and Community Engagement), Stability: International Journal of Security \& Development 3, $\mathrm{n}^{\circ} 1$ (2014) : 14.25.

Gundel, Joakim. Action humanitaire dans le nouvel environnement de sécurité : implications politiques et opérationnelles en Somalie et au Somaliland (Humanitarian Action in the New Security Environment: Policy and Operational Implications in Somalia and Somaliland). Londres : Overseas Development Institute, 2006.

Harroff-Tavel, Marion. Violence et action humanitaire dans les zones urbaines : nouveaux défis, nouvelles approches (Violence and Humanitarian Action in Urban Areas: New Challenges, New Approaches), International Review of the Red Cross 92, $\mathrm{n}^{\circ} 878$ (2010) : $329-50$.

Hazen, Jennifer M. Risque et résilience : comprendre le potentiel de violence (Risk and Resilience: Understanding the Potential for Violence), dans Small Arms Survey 2008, 245-73. Genève : Small Arms Survey, 2008.

Heald, Suzette. Contrôler la criminalité et la corruption d'en bas : les Sungusungu au Kenya (Controlling Crime and Corruption from Below: Sungusungu in Kenya), International Relations 21, $\mathrm{n}^{\circ} 2$ (2007) : 183-99.

Henningsen, Erik, et Peris Jones. "Quel genre d'enfer cela est-il ? » Comprendre la puissance de mobilisation des Mungiki ('What Kind of Hell is This!' Understanding the Mungiki Movement's Power of Mobilisation), Journal of Eastern African Studies 7, $\mathrm{n}^{\circ} 3$ (2013): $371-88$.

Hoddie, Matthew et Caroline A. Hartzell. Renforcer la paix dans les États après la guerre civile : transformer les saboteurs en parties prenantes (Strengthening Peace in Post-Civil War States: Transforming Spoilers into Stakeholders). Chicago et Londres : University of Chicago Press, 2010.

Hoffman, Danny. La signification de la milice : comprendre les forces de défense civile de la Sierra Leone (The Meaning of a Militia: Understanding the Civil Defence Forces of Sierra Leone), African Affairs 106, $\mathrm{n}^{\circ} 425$ (2007) : 639-62.

Hoffmann, Kasper et Koen Vlassenroot. Les groupes armés et l'exercice du pouvoir public : les cas des Mayi-Mayi et des Raya Mutomboki à Kalehe, au Sud-Kivu (Armed Groups and the Exercise of Public Authority: The Cases of the Mayi-Mayi and Raya Mutomboki in Kalehe, South Kivu), Peacebuilding 2, $\mathrm{n}^{\circ} 2$ (2014): -20.

Hofmann, Claudia et Ulrich Schneckener. Engager les acteurs armés non étatiques dans la construction de l'État et de la paix : options et stratégies (Engaging Non-State Armed Actors in State- and Peace-Building: Options and Strategies), International Review of the Red Cross $93, \mathrm{n}^{\circ} 883$ (2011): 603-21.

- - - ONG et acteurs armés non étatiques : améliorer la conformité avec des normes internationales (ONG and Nonstate Armed Actors: Improving Compliance with International Norms). Washington, DC : United States Institute of Peace Press, 2011.

Johnston, Les. Qu'est-ce que l'autodéfense ? (What is Vigilantism?), British Journal of Criminology 36, $\mathrm{n}^{\circ} 2$ (1996) : $220-36$.

Kilcullen, David. La guérilla accidentelle : mener de petites guerres au milieu d'une grosse (The Accidental Guerrilla: Fighting Small Wars in the Midst of a Big One). Oxford : Oxford University Press, 2009.

Kouamé Yao, Séverin. Nouchis, Ziguéhis et Microbes d’Abidjan : déclassement et distinction sociale par la violence de rue en Côte d’Ivoire, Politique Africaine 148, $\mathrm{n}^{\circ} 4$ (2017) : 89-107.

Kriger, Norma. Les politiques du ZANU PF sous le gouvernement de " partage du pouvoir » au Zimbabwe (ZANU PF politics under Zimbabwe's 'PowerSharing' Government), Journal of Contemporary African Studies 30, $\mathrm{n}^{\circ} 1$ (2012) : 11-26.

Lamb, Guy. Militarisation de la police et la guerre contre la criminalité en Afrique du Sud (Police Militarisation and the 'War on Crime' in South Africa), Journal of Southern African Studies 44, $\mathrm{n}^{\circ} 5$ (2018) : 933-49.

Luckham, Robin et Tom Kirk. Les deux faces de la sécurité dans les commandes politiques hybrides : un cadre pour l'analyse et la recherche (The Two Faces of Security in Hybrid Political Orders: A Framework for Analysis and Research), Stability: International Journal of Security \& Development 2, $\mathrm{n}^{\circ} 2$ (2013) : 14. 44

Marshall, Monty G., et Gabrielle C. Elzinga-Marshall. Rapport mondial 2017 : conflit, gouvernance et fragilité de l'État (Global Report 2017 : Conflict, Governance and State Fragility). Vienne, VA : Center for Systemic Peace, 2017.

McLean, Andrew. Sécurité communautaire et cohésion sociale : vers une approche pour le PNUD (Community Security and Social Cohesion: Towards a UNDP Approach). Genève : Programme des Nations Unies pour le développement, 2009.

McQuinn, Brian. DDR et l'organisation interne des groupes armés non étatiques (DDR and the Internal Organization of Non-State Armed Groups), Stability: International Journal of Security \& Development 5, $\mathrm{n}^{\circ} 1$ (2016).

Meagher, Kate. Détournement de la société civile : I'histoire vue de l'intérieur du groupe d'autodéfense les Bakassi Boys dans le sud-est du Nigeria (Hijacking Civil Society: The Inside Story of the Bakassi Boys Vigilante Group of South-eastern Nigeria), The Journal of Modern African Studies 45, $n^{\circ} 1(2007): 89-115$. 
Muggah, Robert. Les Nations Unies ne peuvent pas apporter la paix en République centrafricaine (The U.N. Can't Bring Peace to the Central African Republic), Foreign Policy, 16 août 2018. https://foreignpolicy.com/2018/08/16/the-u-n-cant-bring-peace-to-the-central-african-republic.

Muggah, Robert et Keith Krause. Combler l'écart entre les opérations de paix et l'insécurité post-conflit : vers un programme de réduction de la violence (Closing the Gap Between Peace Operations and Post-Conflict Insecurity: Towards a Violence Reduction Agenda), International Peacekeeping 16, $\mathrm{n}^{\circ} 1$ (2009): 136-50.

Muggah, Robert et Chris O’Donnell. Désarmement, démobilisation et réintégration de prochaine génération (Next Generation Disarmament, Demobilization and Reintegration), Stability: International Journal of Security \& Development 4, $\mathrm{n}^{\circ} 1$ (2015).

Muggah, Robert et Francis Sang. L'ennemi à l'intérieur : repenser la disponibilité des armes en Afrique subsaharienne (The Enemy Within: Rethinking Arms Availability in Sub-Saharan Africa), Conflict, Security \& Development 13, n 4 (2013) : 417-47.

Mutongwizo, Tariro, Lezanne Leoschut et Patrick Burton. Copropriété et collaboration : réflexions sur la mesure des impacts et des changements basées sur les partenariats de prévention de la violence entre les communautés et l'État sur la base de données (Co-ownership and Collaboration: Insights into the Measurement of Impact and Change from Evidence-based Community and State Violence Prevention Partnerships), Stability: International Journal of Security \& Development 4, $\mathrm{n}^{\circ} 1$ (2015).

Newman, Edward. État défaillant et ordre international : construire un monde post-wesphalien (Failed States and International Order: Constructing a PostWestphalian World), Contemporary Security Policy 30, n 3 (2009): 421-43.

- - - Sécurité humaine et constructivisme (Human Security and Constructivism), International Studies Perspectives 2, $\mathrm{n}^{\circ} 3$ (2001) : $239-251$.

Nzekani Zena, Prosper. Les enseignements et les limites du DDR en Afrique (The Lessons and Limits of DDR in Africa), Washington, DC : Africa Center for Strategic Studies, 2013.

Ogada, Mikewa et Njonjo Mue. Réforme du secteur de la sécurité et justice transitionnelle au Kenya (Security Sector Reform and Transitional Justice in Kenya), Nairobi : International Center for Transitional Justice, 2010.

Özerdem, Alpaslan. Une reconceptualisation de la réintégration des anciens combattants : une approche de la « réintégration sociale » (Re-Conceptualisation of Ex-Combatant Reintegration: 'Social Reintegration' Approach), Conflict, Security \& Development 12, $\mathrm{n}^{\circ} 1$ (2012): 51-73.

Petrus, Théodore et Irvin Kinnes. Nouveaux bandits sociaux ? Une analyse comparative du gangstérisme dans les provinces de l'est et de l'ouest du Cap en Afrique du Sud (New Social Bandits? A Comparative Analysis of Gangsterism in the Western and Eastern Cape Provinces of South Africa), Criminology \& Criminal Justice 19, $\mathrm{n}^{\circ} 2$ (2018): 179-96.

Planta, Katrin et Véronique Dudouet. Prêts à négocier ? Options et risques dans la transformation politique des groupes armés non conventionnels (Fit for Negotiation? Options and Risks in the Political Transformation of Non-conventional Armed Groups), Oslo : Centre norvégien de ressources pour la consolidation de la paix (NOREF), 2015.

Podder, Sukanya. De saboteurs à bâtisseurs de l'État : des approches constructives pour l'engagement avec les groupes armés non étatiques dans les États fragiles (From Spoilers to Statebuilders: Constructive Approaches to Engagement with Non-state Armed Groups in Fragile States). Paris : OCDE Publishing, 2012.

Poku, Nana et David T. Graham, eds. Redéfinir la sécurité : mouvements de populations et sécurité nationale (Redefining Security: Population Movements and National Security,). Westport, CT : Greenwood Publishing Group, 1998.

Pottier, Johan. L'ethnographie comme barrière : négocier l'accès humanitaire en Ituri, dans l'est de la RDC (Roadblock Ethnography: Negotiating Humanitarian Access in Ituri, Eastern DR Congo), 1999-2004, Africa: Journal of the International African Institute 76, $\mathrm{n}^{\circ} 2$ (2006) : 151-79.

Pratten, David et Atreyee Sen. Justiciers mondiaux : perspectives sur la justice et la violence (Global Vigilantes: Perspectives on Justice and Violence). Oxford : Oxford University Press, 2007.

Raleigh, Clionadh. Pragmatisme et promiscuité : expliquer la montée de milices politiques compétitives en Afrique (Pragmatic and Promiscuous: Explaining the Rise of Competitive Political Militias across Africa), Journal of Conflict Resolution 60, $\mathrm{n}^{\circ} 2$ (2016) : 283-310.

Risse, Thomas. Gouvernance dans des domaines de statut limité de l'État : introduction et aperçu (Governance in Areas of Limited Stateehood: Introduction and Overview), dans Governance without a State? Policies and Politics in Areas of Limited State Hood, édité par Thomas Risse, 1-35. New York : Columbia University Press, 2011.

Rosenbaum, H. Jon et Peter C. Sederberg. L'autodéfense : une analyse de l'établissement de la violence (Vigilantism: an analysis of establishment violence), Comparative Politics 6, $\mathrm{n}^{\circ} 4$ (1974): 541-70.

Schneckener, Ulrich. Saboteurs ou acteurs de la gouvernance ? Engager les groupes armés non étatiques dans les zones de statut limité de l’État (Spoilers or Governance Actors? Engaging Armed Non-State Groups in Areas of Limited State Hood). SFB - Governance Working Paper Series, ${ }^{\circ}{ }^{2} 1$. Berlin : $^{2}$ DFG Research Center (SFB) 700, 2009.

Schuberth, Moritz. La transformation de la violence politique à la violence criminelle ? Politique, crime organisé et fonctions changeantes dans les groupes urbains armés d'Haïti (A Transformation from Political to Criminal Violence? Politics, Organised Crime and the Shifting Functions of Haiti's Urban Armed Groups), Conflict, Security \& Development 15, n 2 (2015) : 169-96.

- - - Au-delà des trêves des gangs et les politiques de " main de fer » : vers une gouvernance substitutive de la sécurité en Amérique latine (Beyond Gang Truces and Mano Dura Policies: Towards Substitutive Security Governance in Latin America). Stabilité : International Journal of Security \& Development 5, $\mathrm{n}^{\circ} 1$ (2016). 
- - - Remettre en cause l’hypothèse des États faibles : I’autodéfense en Afrique du Sud et au Brésil (Challenging the Weak States Hypothesis: Vigilantism in South Africa and Brazil), Journal of Peace, Conflict \& Development 20 (2013) : 38-51.

- - - L'impact du trafic de drogue sur les acteurs informels de la sécurité dans l'ensemble du Kenya (The Impact of Drug Trafficking on Informal Security Actors in Kenya), Africa Spectrum 49, $\mathrm{n}^{\circ} 3$ (2014) : 55-81.

- - - Désarmement, démobilisation et réintégration dans des contextes non conventionnels : le cas de la réduction de la violence de la MINUSTAH (Disarmament, Demobilisation and Reintegration in Unconventional Settings: The Case of MINUSTAH's Community Violence Reduction), International Peacekeeping 24, $\mathrm{n}^{\circ} 3$ (2017): 410-33.

- - - Faire grandir la base populaire ou soutenir des bandits ? Dilemmes du soutien des donateurs pour la société (non) civile d'Haïti (Growing the Grassroots or Backing Bandits? Dilemmas of Donor Support for Haiti's (Un)Civil Society), Journal of Peacebuilding \& Development 11, $\mathrm{n}^{\circ} 1$ (2016) : 93-98.

- - - Gouvernance hybride de la sécurité, violence post-conflit et légitimité des groupes armés communautaires dans les zones urbaines du Kenya (Hybrid Security Governance, Post-election Violence and the Legitimacy of Community-based Armed Groups in Urban Kenya), Journal of Eastern African Studies 12, $\mathrm{n}^{\circ} 2$ (2018) : 386-404.

- - Le défi des groupes armées communautaires : vers une conceptualisation des milices, des gangs et des justiciers (The Challenge of Communitybased Armed Groups: Towards a Conceptualization of Militias, Gangs, and Vigilantes), Contemporary Security Policy 36, ${ }^{\circ} 2$ (2015): $296-320$.

- - - Engager ou ne pas engager les groupes urbains armés d’Haïti ? Accès sécuritaire aux villes touchées par les catastrophes et les conflits (To Engage or Not to Engage Haiti's Urban Armed Groups? Safe Access in Disaster-stricken and Conflict-affected Cities), Environnement et urbanisation 29, $\mathrm{n}^{\circ} 2$ (2017) : 425-42.

Shaw, Mark et Walter Kemp. Repérer les saboteurs : un guide pour analyser le crime organisé (Spotting the Spoilers: A Guide to Analyzing Organized Crime). New York : International Peace Institute, 2012.

Stankovic, Tatjana et Stina Torjesen. Nouvelles réflexions sur le désarmement, la démobilisation et la réintégration : sondage à l'intention des praticiens (Fresh Insights on Disarmament, Demobilization and Reintegration: A Survey for Practitioners). Oslo : Norwegian Institute of International Affairs, 2010.

Stedman, Stephen John. Problèmes de saboteurs dans les processus de paix (Spoiler Problems in Peace Processes), International Security $22, \mathrm{n}^{\circ} 2$ (1997) : 5-53.

Themnér, Anders. Acte de foi : quand et comment les anciens combattants ont recours à la violence (A Leap of Faith: When and ow Ex-Combatants Resort to Violence), Security Studies 22, n 2 (2013): 295-329.

Torjesen, Stina. Nouvelles avenues pour la recherche et l'étude de DDR (New Avenues for Research in the Study of DDR), Conflict, Security \& Development $9, n^{\circ} 4(2009): 411-23$.

Nations Unies. Guide opérationnel pour des normes intégrées sur le désarmement, la démobilisation et la réintégration (Operational Guide to the Integrated Disarmament, Demobilization and Reintegration Standards), New York : Nations Unies, 2010.

PNUD. Rapport sur le développement humain de 1994 (Human Development Report 1994), New York : Programme des Nations Unies pour le développement (PNUD), 1994.

Verkoren, Willemijn, Rens Willems, Jesper Kleingeld et Hans Rouw. De DDR à la promotion de la sécurité : relier les programmes nationaux aux initiatives communautaires (From DDR to Security Promotion: Connecting National Programs to Community Initiatives), International Journal of Peace Studies $15, \mathrm{n}^{\circ} 2(2010): 1-31$.

Van Metre, Lauren. D’auto-défenseurs à justiciers : un cadre typologique pour les groupes armés communautaires (From Self-Defense to Vigilantism: A Typology Framework of Community-Based Armed Groups). Washington, DC : Réseau RESOLVE, 2019.

Weber, Max. La politique comme vocation (Politics as a vocation), dans From Max Weber: Essays in Sociology, édité par Hans Heinrich Gerth et C. Wright Mills, 77-128. New York : Oxford University Press, 1946.

Yoroms, Gani Joses. Les milices en tant que phénomène social : vers une construction théorique (Militias as a social phenomenon: Towards a theoretical construction), dans Civil Militia: Africa's Intractable Security Menace?, ed. Francis, 31-50. New York, Routledge, 2005.

Young, John. Luttes populaires et cooptation de l'élite : l’Armée blanche de Nuer dans la guerre civile du Sud Soudan (Popular Struggles and Elite Co-optation: The Nuer White Army in South Sudan's Civil War). Genève : Small Arms Survey, 2016.

Zahar, Marie-Joëlle et Delphine Mechoulan. La paix un éléments à la fois ? La médiation locale et la paix durable en République centrafricaine (Peace by Pieces? Local Mediation and Sustainable Peace in the Central African Republic). New York : International Peace Institute, 2017.

Zartman, I. William. Introduction : poser le problème de l'effondrement de l'État (Introduction: Posing the Problem of State Collapse), dans Collapsed States: The Disin-tegration and Restoration of Legitimate Authority, éd. William Zartman, 1-11. Boulder, CO : Lynne Rienner, 1995. 